\title{
Licensing Contracts: Control Rights, Options and Timing
}

\author{
Pascale Crama ${ }^{\dagger}$ \\ Bert De Reyck $^{\ddagger}$ \\ Niyazi Taneri ${ }^{\S}$ \\ ${ }^{\dagger}$ Department of Operations Management, Singapore Management University, \\ pcrama@smu.edu.sg \\ $\ddagger$ UCL School of Management, University College London, bdereyck@ucl.ac.uk \\ $\S$ Engineering Systems \& Design, Singapore University of Technology and Design, \\ niyazitaneri@sutd.edu.sg
}

March 2016 


\title{
The Newsvendor Problem with Advertising Revenue
}

\begin{abstract}
Research and development $(\mathrm{R} \& \mathrm{D})$ collaborations, common in high-tech industries, are challenging to manage due to technical and market risks as well as incentive problems. We investigate how control rights, options, payment terms and timing allow the innovator to capture maximum value from its $R \& D$ collaborations with a marketer. Our study reveals a counterintuitive result; the innovator may, under certain conditions, prefer to grant launch control rights or buy-out options to the marketer despite the fact that both terms restrict its downstream actions. We demonstrate that a menu of contracts is not necessary to address the adverse selection problem as the menu can be replicated by a single option contract. We show that timing, through renegotiation or delayed contracting, as well as the careful allocation of control rights and options can have a significant influence on the value of collaborative R\&D. We provide recommendations on the optimal contract structure and timing based on two project characteristics, novelty of the R\&D process and market-potential variability.

Key words: Research \& Development; Innovation; Contract Design; Moral Hazard
\end{abstract}

\section{Introduction}

Partnerships for research and development (R\&D) projects are common to many researchintensive industries. Such collaborations can improve the value obtained from projects as different partners bring different skills to the partnership. For instance, in the pharmaceutical industry, biotechnology companies often hold innovative product ideas and patents, but lack the financial and marketing capabilities to complete their projects successfully. Conversely, large pharmaceutical firms, who do have those capabilities, are on the lookout for patents they can in-license from biotechnology firms, both because their existing blockbuster patents are on the verge of expiry and due to declining internal R\&D productivity (Pharma Deals Review 2011). In the third quarter of 2014 alone, 125 agreements were signed with a total value exceeding $\$ 11$ billion (Anderson 2014).

Despite their enticing benefits, $R \& D$ collaborations are fraught with uncertainties about the extent of their success. The presence of such uncertainties often results in complex contract structures, such as the inclusion of control rights and options (Lerner and Merges 1998; Elfenbein and Lerner 2003). Both control rights and options allow their holder to react to new information that resolves some of the uncertainty concerning the project. Control 
rights refer to contract clauses that confer the right to make decisions unilaterally about whether and how to pursue further investment in the product. Options may include the right of one partner to buy out the other and take full ownership of the project at a predefined price. Effective licensing contracts should also provide the appropriate incentives for the partners to invest in R\&D and marketing. These investments have a significant impact on the product value but are hard to specify and monitor as they involve highly complex tasks in an uncertain and shifting environment (Alchian and Demsetz 1972, Hart and Moore 1999). Of similar importance is the innovator's decision on when to seek a partner: it impacts both the information available at the time of the agreement and the relative quality of the information available for the two parties involved. As uncertainties are resolved over time, postponing or revisiting the contracting decision may simplify the contract structure and improve performance.

In this paper, we study the effects of contract structure and timing on efforts for and success of R\&D collaborations between two partners who combine different expertise: one partner (the innovator) specializes in $\mathrm{R} \& \mathrm{D}$, whereas the other partner (the marketer) invests exclusively in marketing activities such as advertising and establishing wider distribution networks. This is a stylized form of a situation that often occurs in practice, where an innovator (she) has the technical know-how to create a product, and a marketer (he) brings the product to market. When the product appeals to a niche market and does not require vast distribution networks, an innovator may also be able to commercialize the product on her own, but at a cost disadvantage compared to the marketer, due to lack of experience in marketing. Our study focuses on contract clauses that are often found in R\&D collaboration agreements; the right to decide whether to launch the product or terminate the agreement, as well as a buy-out or buy-back option. For each of these possible contract clauses, we find the payment terms that will maximize the innovator's value. Next, we also study the optimal timing of the contracting decision given this complex contract structure. More specifically, we investigate both contracting at the marketing stage and the case of upfront contracting with downstream renegotiation.

Existing literature has optimized the contractual payment terms - such as fixed fees, royalties and option prices - for different licensor and licensee characteristics and relationships, but always within a pre-specified contract structure. We take an overarching view of the licensing phenomenon and attempt to consolidate and build on existing knowledge by jointly optimizing the licensing contract structure and parameters in a setting with collaborative efforts and a model of the $R \& D$ process reflecting the heterogeneity of $R \& D$ projects. We investigate how an innovator should optimize the payment terms, the allocation of control rights and options and the timing of the contracting decision to capture maximum value 
from the collaboration with the marketer. We identify two factors which should guide both the choice of the contract structure and the timing of the licensing decision: the type of R\&D process and the variability in the product's market potential.

The output of the R\&D process is the extent to which a product is successful, ranging from failure to generate a viable product as the worst-case scenario, to the creation of a mediocre product (low market-potential outcome) or a blockbuster product (high market-potential outcome). We define market-potential variability as the gap between the values of the low and high market-potential outcomes. The size of this gap increases with consumers' sensitivity to product attributes. The higher the sensitivity, the higher the penalty for not meeting expectations on all product attributes. With respect to the type of the R\&D process, we distinguish between novel and incremental processes. When the R\&D process is novel, such as when a company aims to find a new mechanism of action to target a disease, additional R\&D efforts increase the chance of both the low and high market-potential outcomes while reducing the chance of failure. In the example above, these $R \& D$ effort could encompass target identification, lead discovery and optimization as well as the design of appropriate clinical trials. When the $\mathrm{R} \& \mathrm{D}$ is incremental, e.g., R\&D efforts are focused on modifying existing active compounds that work through a known physiological pathway to identify a safer and more effective drug or new disease indications, additional efforts in R\&D increase the probability of a high market-potential outcome while reducing the probability of the low market-potential outcome. Thus in the former case, R\&D effort is more effective at raising the overall probability of success than in the latter.

We find that both the type of the R\&D process and the variability of the market-potential determine which type of control rights and options allow the innovator to extract maximum value from an innovation while partnering with a marketer.

\begin{tabular}{ll|ll}
\hline & & \multicolumn{2}{|c}{ R\&D Process Type } \\
& & Incremental & Novel \\
\hline $\begin{array}{llll}\text { Market-Potential } \\
\text { Variability }\end{array}$ & Low & Delayed/Renegotiation & Innovator launch control \\
& High & Marketer launch control & Buy-out contract \\
\hline
\end{tabular}

Table 1: Optimal contract structure

We now describe some examples of each quadrant in Table $1^{1}$ In 2011, Genentech and FORMA Therapeutics signed an agreement aiming to find a new mechanism of action to

\footnotetext{
${ }^{1}$ Note that some combinations of product characteristics are more likely to occur. For instance, one might expect that novel R\&D is more frequently associated with high market-potential variability (bottom right quadrant) and incremental research with low market-potential variability (top left quadrant).
} 
"starve" tumors, with the stated ambition to address "intractable targets". In this project, the R\&D process entails the identification of suitable molecules that would attach to cancer cells and the subsequent optimization of these molecules while minimizing safety concerns. At the same time, the number of potential targets adds uncertainty about which of them might be successfully addressed and makes market potential more difficult to predict. Together, these factors indicate a novel project with high market-potential variability. The agreement contained a buy-out option for Genentech, allowing it to acquire all rights to the program via a fixed payment.

In the same year, Sam Amer and Ventrus Biosciences signed an agreement to collaborate on the investigation of the efficacy of a known mechanism of action, by adapting drug delivery to and designing clinical tests for an as yet off-label indication with a well-defined market, hemorrhoids - an incremental innovation with low market-potential variability. The royalty rate was renegotiated down at a later date.

Novartis and Paratek Pharmaceuticals' 2009 agreement described drug candidate PTK0796 as "derived from" another class of antibiotics. Thus, R\&D efforts attempt to find the most promising compound in that class that will be safer and more effective than those currently on the market. Despite the incremental nature of the R\&D process, antibiotics face uncertainty about whether the targeted bacteria will develop resistance to existing/competing antibiotics. This leads to high market-potential variability. The agreement was terminated by marketer Novartis, not on technical concerns as demonstrated by the fact that Paratek continued development, indicating a contract with marketer launch control. These contract structures match with the prescriptions of Table 1 .

Lastly, consider the agreement signed between Allos Pharmaceuticals and Mundipharma in 2011. Allos Pharmaceuticals had obtained a fast-track designation for the drug candidate Folotyn pralatrexate prior to the agreement. The Fast Track designation is awarded to "drugs [that] treat serious conditions and fill an unmet medical need". As a drug filling an unmet need, if approved, the drug would face little market-potential variability. R\&D efforts for Folotyn pralatrexate involved a identifying a novel mechanism of action selectively entering cancer cells over-expressed in a certain protein. Although we do not have access to the contract details between Allos and Mundipharma, we posit that it would benefit from innovator launch control based on the project's characteristics. ${ }^{2}$

$R \& D$ process type and market-potential variability affect the optimal contract choice because these two project characteristics interact with the contract structure and payment terms. The innovator exploits these interactions to manage three distinct efficiency losses:

\footnotetext{
${ }^{2}$ We acknowledge that other factors also play a role in the contracting decision which may lead to a different contract structure.
} 
suboptimal R\&D effort, suboptimal marketing effort, and launch by the innovator with higher marketing costs (launch inefficiency). As we will demonstrate, a novel project with low market-potential variability is mainly susceptible to launch and marketing effort inefficiencies. This makes contracts with innovator launch control attractive as the marketer always launches the product and marketing efforts are not distorted by royalties. An incremental project with high market-potential variability is susceptible to R\&D effort inefficiency. Marketer launch control creates incentives for R\&D effort by placing a high prize on the high market-potential outcome. When a project is incremental and has low-market potential variability it is susceptible to all three contract inefficiencies. In such cases, it helps to either delay contracting until more information is available or to renegotiate an upfront contract to avoid inefficiencies. Lastly, a buy-out contract balances effects of all inefficiencies and is suitable for all other R\&D projects.

\section{Literature Review}

We draw on literature from three different areas: the analysis of incomplete contracts in the economics literature, the application of incomplete contracts to collaborative R\&D, and finally, the empirical analysis of R\&D contracts found in the management literature.

We refer to Tirole (1999) for an overview of the state of incomplete contracting research, i.e., contracts cannot contain provisions for all possible future scenarios. He contrasts incomplete and complete contracting, and cautions the researcher about the inherent assumptions of incomplete contracting. Our work is related to a stream of literature devoted to the holdup problem. One approach considers the buyer's and seller's efficient investment and delivery of a good or service. In their model, Hart and Moore (1988) show that, if the court cannot observe why delivery did not take place, the first-best investment levels cannot be achieved except under some restrictive assumptions. Nöldeke and Schmidt (1995), however, show that if the buyer and the seller can sign an option contract giving the buyer the right, but not the obligation to deliver the goods, the first-best investment levels can be achieved. Subsequent articles have shown that the ability of a contract to deliver first-best efficiency may depend, among other things, on the court mechanism used to enforce the contract Edlin and Reichelstein 1996), the nature of the buyer's and the seller's investment (Che and Hausch 1999), risk-aversion (Chung 1991; Holden 1999) and the timing of the investments (Smirnov and Wait 2004).

Grossman and Hart (1986) introduce the notion of control rights as part of a contract, separately from ownership or allocation of returns. When uncontractible elements lead to distortions, an adequate allocation of control rights can reduce the inefficiencies. In Aghion 
and Bolton (1992), first-best efficiency can be achieved through the appropriate allocation of control rights, under certain conditions. Hellmann (1998) studies a venture capital model which shows that an entrepreneur may be willing to concede favorable control terms to the venture capitalist in order to increase his financial reward. We find similar results in a different setting.

The holdup problem has also been investigated in the area of contracting for collaborative investment or research. In a seminal paper on the subject, Aghion and Tirole (1994), posit that careful allocation of ownership and control rights is required in order to create the correct incentives for the researcher. Taking this problem forward, many papers have studied different issues arising in R\&D collaboration by changing the contracting terms or the information structure of the game. Dechenaux et al. (2009) contrast the impact of milestones and royalties on research effort, risk sharing and shelving of projects. Bhaskaran and Krishnan (2009) emphasize the collaborative aspect of new product development (NPD) and propose different mechanisms to share costs or development effort, beyond the incentive effects of milestone and royalty contracts. They find that either cost, effort or no sharing at all can be optimal under different circumstances. Savva and Scholtes (2013) show that research partnerships governed by milestone and royalty contracts lead to inefficient outcomes and describe a contract with opt-out options for either party which restores efficiency. Bhattacharya et al. (2014) similarly find that option contracts can create the correct incentives for partners and achieve risk-sharing. Finally, Xiao and Xu (2012) study the traditional contract structure, but allow for renegotiation of the contract terms. This improves incentives across NPD stages but also increases the cost to the licensee of inducing the innovator to reveal her true value. The main difference with the models studied above is that our setting combines collaborative efforts by both parties under technical uncertainty and market-potential variability with a novel model of R\&D effort impact.

There is a sizable stream of empirical literature studying R\&D licensing contracts confirming that the allocation of control rights is of crucial importance in practice and that the assignment of control rights is closely linked to the financial resources of the R\&D firm (Lerner and Merges 1998) and the relative financial and product market strengths of the alliance partners (Elfenbein and Lerner 2003). In their conclusion, Lerner and Merges (1998) mention that the relationship between the structure of compensation and the allocation of control rights remains to be addressed. Lerner and Malmendier (2010) confirm empirically that when research effort is non-contractible, termination options with (partial) allocation of property rights to the financing entity encourages the innovator to invest adequately in research.

Interest in the timing decision for collaboration on $\mathrm{R} \& \mathrm{D}$ has been rising both in practice 
and in the academic world. In their analysis of the current state of the pharmaceutical industry Kalamas and Pinkus (2003) suggest that the industry would benefit from starting collaborations earlier than it currently does and that better financial terms for small biotechnology firms at earlier stages would motivate biotechnology firms to partner earlier. This would also allow the pharmaceutical partner to avoid more intense competition for late stage licensing opportunities. The literature focuses on firm level and industry level characteristics such as the background of the CEO, prior collaboration experience of the innovator, R\&D intensity of the innovator, introduction of new regulation and patent application and approval as determinants of the timing of the collaboration (Katila and Mang 2003, Gans et al. 2008). In contrast to these studies, our model identifies project level characteristics that have an impact on the timing of an agreement between partners. Thus our paper points to new drivers of licensing contract timing, structure and payment terms, such as the impact of $R \& D$ process type and market-potential variability, that would be interesting to consider in future empirical work.

\section{$3 \quad$ R\&D Collaboration Model}

In this section we define the model characteristics and formulate and solve the first-best project execution (i.e., one from a central planner's perspective).

\subsection{Model Description}

Our model consists of an innovator and a marketer. The innovator owns the intellectual property rights to an innovation and desires to contract with a marketer to bring her innovation to market. The innovator can continue to perform R\&D activities to improve the characteristics of the innovation. The result of this $R \& D$ process is represented in stylized form as three possible outcomes. First, if the technical features do not exceed a minimum threshold, the product cannot be launched. Second, the product could have low marketpotential. Third, the product could have high market-potential. Thus, our model allows us to go beyond a binary outcome of failure or success. Different market-potential values could result from the specific combination of technical features that may affect the product's sales prospects, for instance the efficacy and side effects of a new drug, or the weight, dimension and battery life for electronics. We express market-potential as a scalar measure that summarizes the base sales prospects of the product. Product sales, however, not only depend on the market-potential, but also on how successfully the product is marketed. The innovator

has the necessary know-how to complete R\&D, but she may lack the experience and the resources for a successful product launch. The marketer, on the other hand, has expertise 
in new product management and desires to contract with the innovator to add innovative products to his product portfolio.

R\&D collaboration contracts can contain the following commonly found payment terms: a contract signature fee, a milestone payment at successful completion of the research phase, and a royalty percentage on the sales. Most R\&D collaboration contracts will also specify non-monetary clauses that determine the rights and obligations of both parties within the relationship. We focus on the right (a) of both parties to choose whether to launch the product, (b) for the marketer to buy out the innovator from the contract and (c) for the innovator to buy back the product.

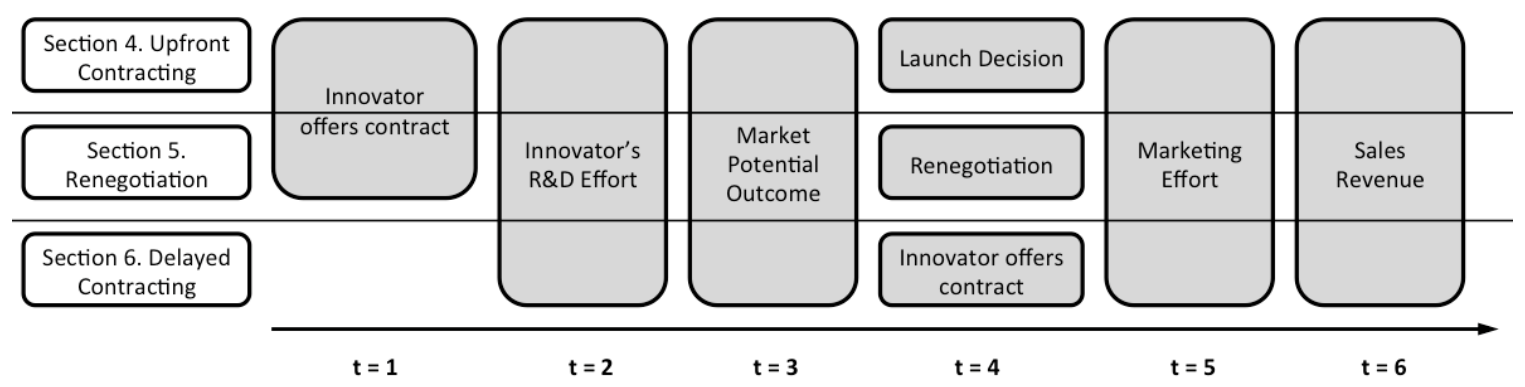

Figure 1: Project and Contract Timeline

The timeline of the project and the interaction between the innovator and the marketer is given in Figure 1. We have created a separate timeline for different timing scenarios: upfront contracting, upfront contracting with renegotiation, and delayed contracting. At time $t=1$, the innovator negotiates and signs a contract with the marketer or decides to delay contracting until $t=4$. The contract specifies the payments from the marketer to the innovator (upfront payment $s$, milestone payment $l$, and royalty rate $r$ ) and can also include clauses allocating control rights pertaining to product launch, or buy-out and buy-back options. The innovator decides on her optimal R\&D effort level, $e$, and incurs a corresponding cost, $c e^{2} / 2$, at $t=2$. This influences the R\&D outcome of the product, which is observed at $t=3$. The outcome can be failure (insufficient for launch), low or high market-potential $\left(\alpha_{0}, \alpha_{L}\right.$ and $\alpha_{H}$ respectively). The R\&D effort influences the likelihood of the three outcomes $\left(\pi_{i}(e)=p_{i}+\gamma_{i} e, i \in\{0, L, H\}\right)$ and has a positive effect on expected market-potential. At $t=4$, one of three events can take place. If an upfront contract was signed, either a launch decision has to be taken or the contract can be renegotiated. If the decision was to delay at time $t=1$, contract negotiation takes place for technically successful products. After the product is launched, the marketer, or possibly the innovator if the marketer chooses not to launch when the market-potential outcome is low, decides on the appropriate marketing effort at $t=5\left(\mu_{i M}\right.$ for the marketer or $\mu_{L I}$ for the innovator) 
and incurs the cost of effort $\left(k_{j} \mu_{i j}^{2} / 2, j \in\{M, I\}\right)$. Sales revenues at $t=6$ are a function of market-potential and marketing effort $\left(v\left(\alpha_{i}, \mu_{i j}\right)=\alpha_{i} \mu_{i j}\right)$. We assume that the innovator and marketer are rational, risk-neutral agents who choose their effort levels to maximize respective individual profit.

Central to the contracting problem is the assumption that the innovator's and marketer's effort levels are unobservable, and thus cannot be meaningfully written into a contract. While it would be possible to specify the amount of resources to be invested, we argue that it is unrealistic to define $R \& D$ or marketing input in terms of resources spent, because the quality of the effort influences the impact of invested resources. Because the effort level of the innovator and marketer are both unobservable, we deal with a two-sided moral hazard problem and the contract will have to offer incentives to both parties to invest an appropriate level of effort.

We summarize our notation in Table 2 and provide an explanation below. To guarantee

\begin{tabular}{llll}
\hline & \multicolumn{2}{c}{ Decisions } & \multicolumn{1}{c}{ Parameters } \\
\hline$e$ & Innovator's R\&D effort & $\alpha_{i}$ & Product's market-potential \\
$\mu_{L I}$ & Innovator's marketing effort in & $\gamma_{i}$ & Impact of R\&D effort on probability of $\alpha_{i}$ \\
& product of market-potential L & $p_{i}$ & Probability of R\&D outcome without effort \\
$s$ & Upfront payment at signing & $\pi_{i}(e)$ & Probability of R\&D outcome with effort $e$ \\
$l$ & Milestone payment at launch & $v\left(\alpha_{i}, \mu_{i j}\right)$ & Sales value function \\
$r$ & Royalty rate on sales & $c$ & Innovator's R\&D effort cost factor \\
$\mu_{i M}$ & Marketer's marketing effort in & $k_{I}$ & Innovator's marketing effort cost factor \\
& product of market-potential $i$ & $k_{M}$ & Marketer's marketing effort cost factor \\
& \multirow{2}{*}{$* i \in\{0, H, L\}$ indicates R\&D outcomes (market-potential) and $j \in\{I, M\}$ indicates player } \\
\hline
\end{tabular}

Table 2: Notation used in models

concavity of the objective function, we made the following assumptions: the probabilities of the three scenarios of market-potential are linear in R\&D effort, sales revenue is linear in marketing effort, and the cost of research and marketing is quadratic in effort. We assume that blockbuster products can only be successfully marketed by the marketer, with a cost factor $k_{M}$, whereas the innovator can potentially market a low market-potential product herself, with a cost factor $k_{I}$, and $k_{I}>k_{M}$. To reduce notation, we set $c=1, p_{H}=0$ and write $\pi_{0}(e)=1-\pi_{L}(e)-\pi_{H}(e)$, with $\gamma_{H}=1$ (wlog). This allows us to drop the subscript for $\gamma$ in the probability functions and write $\pi_{L}=p_{L}+\gamma e$ and $\pi_{H}=e$. We allow $\gamma \in[\underline{\gamma}, \bar{\gamma}] \in[-1,1]$. The limits $\underline{\gamma}$ and $\bar{\gamma}$ are chosen to ensure that $\pi_{i}$ remains a valid probability distribution (the limits will be explicitly calculated after the model exposition) $3^{3}$

\footnotetext{
${ }_{3}^{3}$ Schuett $(2012)$ also adopts a model where effort influences types of outcomes for a firm licensing out a
} 
Another important factor to consider is the nature of the R\&D process under consideration: different projects are affected differently by the R\&D effort expended on them. In particular, for a novel R\&D process, research effort increases the probability of being successful (reduces $\pi_{0}$ ), more than it distinguishes between high and low market-potential outcomes. This implies a $\gamma$ close to 1 . In the pharmaceutical industry, this could correspond to a project aimed at the discovery of an entirely new mechanism of action against a particular disease. Efforts of the company in trying to develop this new mechanism of action may reduce the chance of failure but increase the probability of both high and low market-potential outcomes to a similar degree. At the other extreme, we have research that is incremental in nature and alters features of an existing product or applies a known technology to a new market. In that case, the R\&D effort does not so much increase the probability of success as affect the relative probabilities of market-potential outcomes (increases $\pi_{H}$ mostly at the expense of $\left.\pi_{L}\right)$. This corresponds to $\gamma$ close to -1 . We can illustrate this category with the development of a fixed-dose or single-tablet regimen of an existing drug with a proven mechanism of action where higher R\&D effort would improve compliance and hence adoption, while technical success is relatively assured regardless of the $R \& D$ effort. By varying the parameter $\gamma$ from -1 to 1 , we allow for intermediate scenarios of $R \& D$ effort impact.

Another important assumption is that the achieved market-potential (upon observation of technical success) is non-verifiable, i.e., cannot be be contracted upon. 4 This implies that (a) the milestone payment cannot vary depending on the market-potential and (b) the milestone payment is conditioned on the launch. Similar assumptions for the definition of a milestone payment are made in Savva and Scholtes (2013) and Bhattacharya et al. (2014). This is in line with management practice, where future market-potential is very hard to contract upon.

\subsection{Social Optimum}

We determine the socially optimal execution (first-best execution) of the collaboration, which will serve as a benchmark for the performance of a contractual relationship. In this case, the decision rights are given to a central planner who determines the research and marketing

patent for two fields of use. In the absence of overlap between fields of use, licensees act as monopolies. With overlap, licensees compete, which reduces value. Higher effort reduces the chance of overlap. In contrast, our model considers high-low outcomes along with a failure outcome and distinguishes between different types of R\&D processes.

${ }^{4}$ While contracts often have financial terms that vary with the level of sales achieved, such as sales milestones, royalties or tiered royalties, these payments depend on the actual sales realized, not the underlying market-potential. In other words, whether a given sales level has been reached with lower market-potential and high effort or higher market-potential but lower effort cannot be verified. Therefore, one cannot contract upon market-potential directly. 
efforts. The following formulation optimizes the expected total project value $V^{S O C}$ :

$$
\max _{e \geq 0, \mu_{i M} \geq 0} V=e\left(\alpha_{H} \mu_{H M}-\frac{k_{M} \mu_{H M}^{2}}{2}\right)+\left(p_{L}+\gamma e\right)\left(\alpha_{L} \mu_{L M}-\frac{k_{M} \mu_{L M}^{2}}{2}\right)-\frac{e^{2}}{2}
$$

resulting in: $\quad \mu_{i M}^{S O C}=\frac{\alpha_{i}}{k_{M}}$,

$$
\begin{aligned}
e^{S O C} & =\frac{\gamma \alpha_{L}^{2}+\alpha_{H}^{2}}{2 k_{M}}, \\
V^{S O C} & =\frac{p_{L} \alpha_{L}^{2}}{2 k_{M}}+\frac{\left(\gamma \alpha_{L}^{2}+\alpha_{H}^{2}\right)^{2}}{8 k_{M}^{2}} .
\end{aligned}
$$

The central planner always assigns the task of marketing the product to the marketer as he has a lower marketing effort cost. The marketing effort decision can be separated from the R\&D effort decision as the optimal marketing effort depends only on the product's realized market-potential and not on the $R \& D$ effort preceding it. The optimal R\&D effort, $e^{S O C}$, depends on the marketing effort, as the latter determines the sales revenue under each outcome. ${ }^{5}$ Self-interested innovators and marketers, however, deviate from socially optimal effort levels and the contract must align incentives to achieve maximum value.

We develop a series of models with different contract structures, terms and timings in the remainder of the paper (please refer to Figure 1 for an overview of the different models covered). In these models, we take the perspective of the innovator who owns the invention. We therefore use the terms optimum/optimal to refer to the innovator's payoff and the terms social optimum/socially optimal as they were used in this subsection.

\section{$4 \quad$ Upfront Contracting}

Innovators often contract with the marketer before the R\&D effort has been fully completed. In that case, a significant portion of the technical and commercial risk is still remaining. The contract structure should therefore include clauses that allow the partnership to react to the resolution of the uncertainties, such as through the allocation of launch control or options.

For analytical tractability we start out by limiting our contracts to two payment terms. The milestone contract contains an upfront payment and a milestone payment at product launch. The royalty contract contains an upfront payment and a percentage royalty on product sales. These simple contracts allow us to focus on the underlying interactions between payment terms and structural elements. Milestone payments affect the desirability of launch from the marketer's perspective which makes launch control an effective value creation mechanism. Conversely, royalties create an efficiency loss by distorting the marketer's effort and

\footnotetext{
${ }^{5}$ The definition of $e^{S O C}$ allows us to determine $\bar{\gamma}=\min \left\{\gamma^{*}, 1\right\}$, with $\gamma^{*}$ such that $(1+\gamma) e^{S O C}=1-p_{L}$.
} 
options can be used to address this problem. We first analyze control rights paired with milestone contracts, then turn to options paired with royalty contracts. We also confirm numerically that our results hold for contracts that include all three payment terms.

\subsection{Launch Control}

First, we focus on the allocation of the right to decide whether or not to launch the product to either the innovator or the marketer. Intuitively, we can immediately discern that the innovator's and the marketer's decisions will not always be aligned: the innovator is always eager to launch, as she no longer bears any costs once her part of the development phase is completed, whereas the marketer will only want to launch if the product will be sufficiently profitable to cover the contractual payments to the innovator.

Launch control is particularly relevant in milestone payment contracts, in which the marketer agrees to pay the innovator an upfront payment $s$ and a milestone payment $l$. Milestone contracts offer the advantage of not distorting the effort level of the marketer: a milestone payment is constant regardless of the marketer's effort whereas under a royalty rate, an increase in the marketer's effort increases the royalty payments he has to make. A high milestone payment, however, may turn a marginally profitable invention into a loss to the marketer, and the marketer and the innovator may disagree on launching the invention.$^{6}$ Thus we focus on milestone payment contracts with first the innovator and then the marketer holding the launch control rights.

\subsubsection{Innovator Launch Control}

As mentioned earlier, the innovator always benefits from launch as she earns a milestone payment $l$ and bears no further cost. The innovator's optimization problem, taking into account the marketer's incentive and participation constraints, is given by:

$$
\begin{gathered}
\max _{s \geq 0, l \geq 0} \hat{e} l+\left(p_{L}+\gamma \hat{e}\right) l-\frac{\hat{e}^{2}}{2}+s \\
\text { s.t. } \quad \hat{\mu}_{i M}={ }_{\mu_{i M} \geq 0}\left\{\alpha_{i} \mu_{i M}-\frac{k_{M} \mu_{i M}^{2}}{2}-l\right\}, \quad i=\{H, L\} \\
\hat{e}=(\gamma+1) l \\
\hat{e}\left(\alpha_{H} \hat{\mu}_{H M}-\frac{k_{M} \hat{\mu}_{H M}^{2}}{2}-l\right)+\left(p_{L}+\gamma \hat{e}\right)\left(\alpha_{L} \hat{\mu}_{L M}-\frac{k_{M} \hat{\mu}_{L M}^{2}}{2}-l\right)-s \geq 0
\end{gathered}
$$

\footnotetext{
${ }^{6}$ This will not happen with a royalty-only contract, as the payment to the innovator is proportional to the revenue made by the marketer, and in a low realization, the lower royalty payment to the innovator guarantees that the product remains profitable to the marketer who will want to launch the product regardless of the technical outcome.
} 
Eq (6) reflects the marketer's optimization problem for the marketing effort. Eq (7) determines the innovator's optimal R\&D effort. Finally, Eq (8) ensures that the marketer obtains his reservation utility, which is set to zero.

Theorem 1 A milestone contract with innovator launch control achieves the social optimum if and only if $p_{L}=0$ and $\gamma \geq 0$. In that case, the optimal contract is $(s, l)=$ $\left(0, \frac{\alpha_{H}^{2}+\alpha_{L}^{2} \gamma}{2(1+\gamma) k_{M}}\right)$. If $p_{L}>0$, the social optimum is not achieved and the optimal contract is $(s, l)=\left(0, \frac{\left(\alpha_{H}^{2}+\gamma \alpha_{L}^{2}\right)(1+\gamma)-2 k_{M} p_{L}+\sqrt{\left(\left(\alpha_{H}^{2}+\gamma \alpha_{L}^{2}\right)(1+\gamma)-2 k_{M} p_{L}\right)^{2}+8 k_{M}(1+\gamma)^{2} \alpha_{L}^{2} p_{L}}}{4 k_{M}(1+\gamma)^{2}}\right)$.

All proofs are in the Appendix. This theorem tells us that us that milestone payments may not be effective for every $R \& D$ process type. When $\gamma=-1$, a milestone contract fails to induce any R\&D effort at all; because the milestone payment does not depend on the achieved market-potential and effort does not increase the chances of technical success, the innovator has no incentive to expend any effort. As $\gamma$ varies from -1 to 1 , ceteris paribus, the innovator's value from milestone contracts will increase, as milestone payments will become increasingly effective at creating an incentive for the innovator, and might even be able to achieve social optimum under the condition listed in Theorem 1 .

\subsubsection{Marketer Launch Control}

Allocating launch control to the marketer creates a new constraint for the innovator: once the market-potential is revealed, the marketer will only want to launch if his profit, net of the milestone payment to the innovator, is positive. Therefore, if the innovator designs the contract to induce the marketer to launch the product in both market-potential outcomes $(\delta=1)$, the milestone payment is bounded by the profit under the low market-potential outcome, and the innovator's incentive to invest in research will be correspondingly low. If the milestone payment exceeds the net profit under the low market-potential outcome, the marketer will prefer not to launch the low market-potential product $(\delta=0)$ and only launch the high market-potential product. We call such a contract an exclusion contract. If the marketer refuses to launch the product, the innovator regains all rights to the innovation and can launch the product on her own. We modify the innovator's optimization problem accordingly.

$$
\max _{s \geq 0, l \geq 0} \hat{e} l+\hat{\delta}\left(p_{L}+\gamma \hat{e}\right) l+(1-\hat{\delta})\left(p_{L}+\gamma \hat{e}\right)\left(\alpha_{L} \hat{\mu}_{L I}-\frac{k_{I} \hat{\mu}_{L I}^{2}}{2}\right)-\frac{\hat{e}^{2}}{2}+s
$$




$$
\begin{aligned}
& \text { s.t. } \hat{\mu}_{i M}={ }_{\mu_{i M} \geq 0}\left\{\alpha_{i} \mu_{i M}-\frac{k_{M} \mu_{i M}^{2}}{2}-l\right\}, \quad i=\{H, L\} \\
& \hat{\delta}={ }_{\delta=\{0,1\}}\left\{\delta\left(\alpha_{L} \hat{\mu}_{L M}-\frac{k_{M} \hat{\mu}_{L M}^{2}}{2}-l\right)\right\} \\
& \hat{\mu}_{L I}={ }_{\mu_{L I} \geq 0}\left\{\alpha_{L} \mu_{L I}-\frac{k_{I} \mu_{L I}^{2}}{2}\right\} \\
& \hat{e}=(\hat{\delta} \gamma+1) l+(1-\hat{\delta}) \gamma \frac{\alpha_{L}^{2}}{2 k_{I}} \\
& \hat{e}\left(\alpha_{H} \hat{\mu}_{H M}-\frac{k_{M} \hat{\mu}_{H M}^{2}}{2}-l\right)+\hat{\delta}\left(p_{L}+\gamma \hat{e}\right)\left(\alpha_{L} \hat{\mu}_{L M}-\frac{k_{M} \hat{\mu}_{L M}^{2}}{2}-l\right)-s \geq 0
\end{aligned}
$$

Theorem 2 There exists a $\hat{\gamma} \in[-1,1]$ such that for all $\gamma \geq \hat{\gamma}$ the optimal contract is $(s, l)=\left(\frac{\left(\alpha_{H}^{2}-\alpha_{L}^{2}\right) \alpha_{L}^{2}(1+\gamma)}{4 c k_{M}^{2}}, \frac{\alpha_{L}^{2}}{2 k_{M}}\right)$ with the project being launched by the marketer under both market-potential outcomes, whereas for all $\gamma<\hat{\gamma}$, an exclusion contract $(s, l)=\left(0, \frac{\alpha_{H}^{2}}{2 k_{M}}\right)$ is optimal.

Theorem 2 shows that it may not always be optimal for the innovator to aim for both market-potential outcomes to be launched by the marketer, despite the marketer having a lower cost of marketing effort. To guarantee the marketer's launch of the low marketpotential product, the milestone payment is restricted by his profit in that scenario. As the milestone payment $l$ is constant and does not reflect the higher value obtained in case of a high market-potential outcome, the innovator has a relatively low incentive to invest in R\&D to achieve a high market-potential outcome. This constraint on $l$ is particularly restrictive if the market-potential variability is large. The problem is compounded for low $\gamma$ as the R\&D effort increases the probability of a high market-potential outcome at the expense of the low market-potential outcome, and the ideal R\&D effort can only be induced by giving strong incentives linked to the high market-potential outcome. In such cases, it might be more profitable for the innovator to create a strong incentive to invest in research by setting the milestone payment such that the marketer will only launch the high market-potential outcome.7

Intuitively, we expect that giving away control to the marketer should not be in the innovator's interest as it adds a constraint to her decision problem. Furthermore, as the marketer enjoys a cost advantage in marketing effort, an exclusion contract that forces the innovator to launch the low market-potential outcome herself is inefficient, and innovator launch control would seem more appropriate. Nonetheless, we find that it can be in the innovator's interest to yield control over the launch decision to the marketer when an exclusion contract is optimal. We formally characterize the optimal contract in the following theorem.

\footnotetext{
${ }^{7}$ The case of the exclusion contract, by achieving a high $\mathrm{R} \& \mathrm{D}$ effort when $\gamma<0$, allows us to determine $\underline{\gamma}=\max \left\{-1, \gamma^{*}\right\}$, with $\gamma^{*}$ such that $-\gamma\left(\alpha_{H}^{2} /\left(2 k_{M}\right)+\gamma \alpha_{L}^{2} /\left(2 k_{I}\right)\right)=p_{L}$.
} 
Theorem 3 If $\hat{\gamma}>-1$, then a $\tilde{\gamma} \leq \hat{\gamma}$ exists such that for all $\gamma \leq \tilde{\gamma}$ an exclusion contract is more profitable than the optimal value from a milestone contract with innovator launch control.

Ceding control is unattractive for $\gamma \geq \hat{\gamma}$, when the optimal contract under marketer launch control is to launch both outcomes: the launch decision remains the same, but a constraint on the milestone payment is added. However, if a region exists in which the exclusion contract is optimal, i.e., $\hat{\gamma}>-1$, there will be a region such that ceding launch control is optimal for $-1 \leq \gamma \leq \tilde{\gamma} \leq \hat{\gamma}$.

The implications of Theorem 3 may be more apparent when we investigate Figure 2. In general, which contract is preferred depends on trade-offs made between various inefficiencies caused by each contract. First, note that exclusion contracts create a launch inefficiency for low market-potential products because the product is launched by the innovator who is at a cost disadvantage when it comes to exerting marketing effort. This launch inefficiency is more pronounced when $\alpha_{L}$ is high, i.e. variability is low. When this is the case, it is favorable to have innovator launch control which avoids the launch inefficiency and ensures that the product is always launched by the marketer who has a cost advantage. Second, the fact that the marketer only launches the high market-potential product means that the innovator can charge a very high milestone payment when signing an exclusion contract. This high milestone payment, in turn, creates incentives for the innovator to increase the probability of the high market-potential outcome by increasing her R\&D effort. When $\gamma$ is low, increased R\&D effort also translates into a lower chance of suffering from the launch inefficiency, further increasing the attractiveness of the exclusion contract. Combining these two effects, we can see that lower $\gamma$ and higher variability make exclusion contracts attractive while higher $\gamma$ and lower variability make innovator launch control more attractive. In summary, while an exclusion contract may substantially reduce the profit under the low market-potential outcome, it allows a high milestone payment which creates an incentive for higher R\&D effort. As the innovator cannot credibly commit to not launching once the market-potential has been revealed to be low, she creates commitment by ceding launch control to the marketer.

\subsection{Options}

Another way to govern the launch of a product is to grant buy-out or buy-back options. Under royalty contracts, options rather than control rights become valuable tools because the launch decision will be unanimous. Royalty contracts offer two advantages: they offer the innovator an incentive that is directly proportional to the value created by the R\&D effort and 


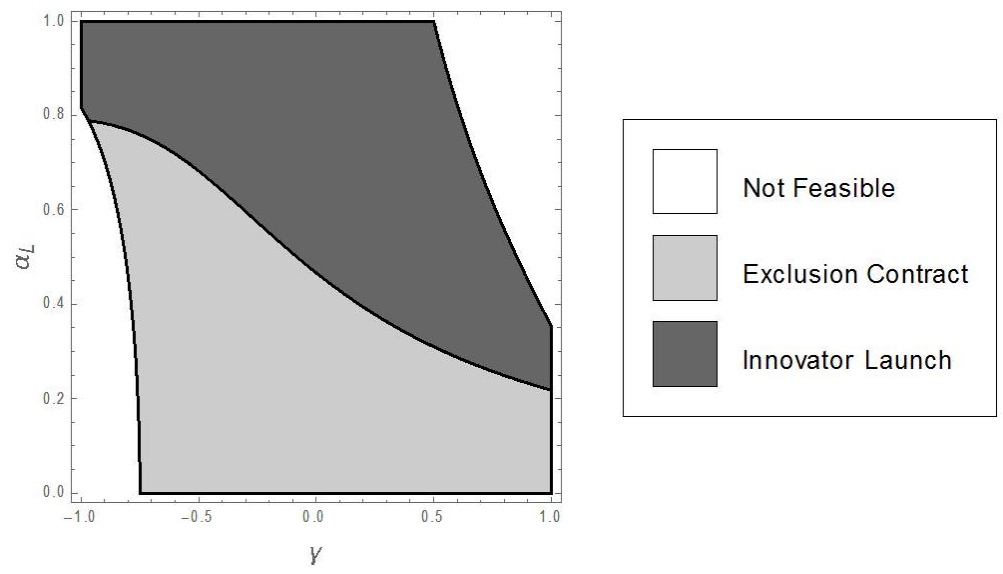

Figure 2: Optimal milestone contracts: innovator vs marketer launch control

$$
\left(\alpha_{H}=1, k_{M}=1.5, k_{I}=4, p_{L}=0.25\right)
$$

they do not distort the launch decision. In our model without fixed costs related to product launch, there is no distortion of the launch decision and the low and high market-potential outcomes are both profitable to the marketer. Therefore, we do not differentiate between innovator and marketer launch control. However, royalties present the major drawback of distorting marketer effort, destroying a fraction of the project's realized value. This also means that royalties are most damaging for high market-potential realizations. This quandary makes buy-out options attractive 8

A buy-out option can be beneficial if the innovator can set a high strike price that the marketer wishes to exercise in the high market-potential outcome, without preventing him from launching the product under the low market-potential outcome. We find that buyback options on their own are never optimal as an exclusion contract can be used to ensure innovator launch of the low market-potential outcome. Finally, it is possible to combine buyout and buy-back options in one contract. This contract, however, will simplify to either the buy-out or the exclusion contract, depending on which contract structure is optimal.

\subsubsection{Buy-Out Option}

A contract can offer a buy-out option to the marketer by specifying the strike price of the option that he has to pay to the innovator, and the time at which it can be exercised. In our timeline, the option expiry is logically set after the R\&D outcome is revealed but before the launch decision is taken. As a benchmark, we will first determine the optimal royalty rate without a buy-out option, and then discuss the impact of a buy-out option.

Optimal royalty rate without buy-out option A simple royalty contract without a

\footnotetext{
${ }^{8}$ Buy-out option would be redundant in combination with a milestone contract in which the buy-out price would be equal to the milestone payment.
} 
buy-out option leads to the following optimization problem.

$$
\begin{gathered}
\max _{s \geq 0,0 \leq r \leq 1} \hat{e} r \alpha_{H} \hat{\mu}_{H M}+\left(p_{L}+\gamma \hat{e}\right) r \alpha_{L} \hat{\mu}_{L M}-\frac{\hat{e}^{2}}{2}+s \\
\text { s.t. } \hat{\mu}_{i M}={ }_{\mu_{i M} \geq 0}\left\{(1-r) \alpha_{i} \mu_{i M}-\frac{k_{M} \mu_{i M}^{2}}{2}\right\}, \quad i=\{H, L\} \\
\hat{e}=\left(\gamma \alpha_{L} \hat{\mu}_{L M}+\alpha_{H} \hat{\mu}_{H M}\right) r \\
\hat{e}\left((1-r) \alpha_{H} \hat{\mu}_{H M}-\frac{k_{M} \hat{\mu}_{H M}^{2}}{2}\right)+\left(p_{L}+\gamma \hat{e}\right)\left((1-r) \alpha_{L} \hat{\mu}_{L M}-\frac{k_{M} \hat{\mu}_{L M}^{2}}{2}\right)-s \geq 0
\end{gathered}
$$

Proposition 1 If $p_{L}=0$, then the optimal royalty rate is a constant $r^{*}=1 / 3$. If $p_{L}>0$, then the optimal royalty rate drops below $1 / 3$.

The optimal royalty rate balances the detrimental effect on value due to the reduced marketing effort with the increase in probability of launch achieved by giving an incentive to the innovator to invest in $\mathrm{R} \& \mathrm{D}$ effort. A higher $p_{L}$ decreases the optimal royalty rate because the need for R\&D effort is less and the detrimental impact on value after launch gains in importance.

Buy-out option and royalty rate The buy-out clause specifies a price $B$ at which the marketer can buy out the innovator after the $R \& D$ outcome has been revealed. If the marketer exercises the buy-out clause, he no longer pays the royalty on the product sales, and he will invest the socially optimal amount of marketing effort because he becomes the sole owner of the product. Clearly, this is most valuable if the option is exercised for the high market-potential outcome, as the losses from the distortion due to the royalty rate are largest. The contract should be structured such that it creates discrimination between the low and high market-potential outcomes without requiring external enforcement. This is achieved by setting the buy-out price $B$ such that:

$$
\left\{\begin{array}{l}
\frac{\alpha_{H}^{2}}{2 k_{M}}-B \geq \frac{(1-r)^{2} \alpha_{H}^{2}}{2 k_{M}} \quad \Longleftrightarrow \quad B \leq \frac{r(2-r) \alpha_{H}^{2}}{2 k_{M}} \\
\frac{\alpha_{L}^{2}}{2 k_{M}}-B \leq \frac{(1-r)^{2} \alpha_{L}^{2}}{2 k_{M}} \Longleftrightarrow B \geq \frac{r(2-r) \alpha_{L}^{2}}{2 k_{M}}
\end{array}\right.
$$

The innovator prefers the highest possible exercise price and sets $B=r(2-r) \alpha_{H}^{2} /\left(2 k_{M}\right)$, and the marketer will not exercise the buy-out option under the low market-potential outcome. Thus we formulate the innovator's optimization problem as follows: $\mathrm{9}^{9}$

$$
\max _{s \geq 0,0 \leq r \leq 1} \hat{e} \frac{r(2-r) \alpha_{H}^{2}}{2 k_{M}}+\left(p_{L}+\gamma \hat{e}\right) r \alpha_{L} \hat{\mu}_{L M}-\frac{\hat{e}^{2}}{2}+s
$$

\footnotetext{
$B$.

${ }^{9}$ The definition of $\hat{\mu}_{H M}$ and $B=r(2-r) \alpha_{H}^{2} /\left(2 k_{M}\right)$ implies that $(1-r) \alpha_{H} \hat{\mu}_{H M}-k_{M} \hat{\mu}_{H M}^{2} / 2=\alpha_{H}^{2} /\left(2 k_{M}\right)-$
} 


$$
\begin{aligned}
\text { s.t. } \quad \hat{\mu}_{i M} & ={ }_{\mu_{i M} \geq 0}\left\{(1-r) \alpha_{i} \mu_{i M}-\frac{k_{M} \mu_{i M}^{2}}{2}\right\}, \quad i=\{H, L\} \\
\hat{e} & =\left(\gamma \alpha_{L} \hat{\mu}_{L M}+\frac{(2-r) \alpha_{H}^{2}}{2 k_{M}}\right) r \\
& \hat{e}\left((1-r) \alpha_{H} \hat{\mu}_{H M}-\frac{k_{M} \hat{\mu}_{H M}^{2}}{2}\right)+\left(p_{L}+\gamma \hat{e}\right)\left((1-r) \alpha_{L} \hat{\mu}_{L M}-\frac{k_{M} \hat{\mu}_{L M}^{2}}{2}\right)-s \geq 0
\end{aligned}
$$

Theorem 4 A royalty contract with buy-out option yields a higher expected value to the innovator than a royalty contract without buy-out option. Furthermore, the royalty rate of a buy-out contract exceeds the royalty rate of a royalty contract.

This theorem shows that buy-out options are relevant in the presence of royalties as they allow the innovator to increase the royalty rate — with positive effects on R\&D effort yet limits the adverse effect of royalties to the low market-potential outcome. While it is generally accepted that holding an option usually creates value, the theorem demonstrates that giving away an option is also valuable when it creates a credible commitment to higher R\&D effort.

Our prior analysis revealed that it could be optimal to assign launch control rights to the marketer in order to credibly signal high $R \& D$ effort. This came at the cost of the innovator having to launch the low market-potential products, despite her cost disadvantage. A buyout contract offers a solution to the launch inefficiency: the increased royalty rate does not prevent launch of the low market-potential outcome but acts as a motivation for the marketer to exercise the buy-out option under the high market-potential outcome. Thus the incentive to invest in research is still strong as the innovator hopes to achieve the higher prize of the buy-out option, without forcing launch inefficiency. Whether this creates a gain over the exclusion contract depends on the comparison of two inefficiencies: namely, the higher marketing cost of the innovator versus the negative impact of the royalty rate on the marketer's effort. As the comparison between milestone contracts with innovator launch control and royalty contracts with buy-out options is inconclusive, we resort to numerical methods to determine which type of contract is optimal for different problem paremeters.

\subsubsection{Buy-Back Option}

In a setting where the innovator has the option to buy back her product with no option assigned to the marketer, we find that exclusion contracts render contracts with buy-back options suboptimal. By setting a high enough milestone, the exclusion contract allows the innovator to take back its product for free while also avoiding sales-distorting royalties. The result regarding two-way options below will make this outcome more apparent. 


\subsubsection{Two-Way Option}

Buy-out and buy-back options can be included in a single contract where the two parties potentially exercise different options in different scenarios. However, we find that such a contract is equivalent to either a buy-out option or an exclusion contract.

Theorem 5 In a two-way option contract, the innovator either sets an arbitrarily high buyback price making it suboptimal to ever exercise the buy-back option, or sets $r=1$, the buy-back strike price to zero and the buy-out strike price to $\alpha_{H}^{2} /\left(2 k_{M}\right)$.

In the first case of Theorem 5, the innovator never exercises the buy-back option which makes the two-way option equivalent to a buy-out option. In the latter case, a two-way option contract allows an option to be exercised in both market-potential outcomes. This, in turn, allows the innovator to set royalties to $r=1$ without harming marketing efforts because royalties will always be avoided due to the exercised options. As royalties are set to 1, the product is worthless in the hands of the marketer, and he is willing to allow the innovator to "buy" the product back for free. With $r=1$, the buy-out strike price $r(2-r) \alpha_{H}^{2} /\left(2 k_{M}\right)$ becomes $\alpha_{H}^{2} /\left(2 k_{M}\right)$, which makes the contract equivalent to an exclusion contract.

Figure 3 compares the values of three different contracts. The optimal contract choice depends on the tradeoffs between the launch, marketing and research inefficiencies created by each contract structure. For novel projects (high $\gamma$ ) and/or low market-potential variability (high $\alpha_{L}$ ), launch and marketing inefficiencies are costly and a milestone contract with innovator launch control is preferable. For incremental products (low $\gamma$ ), research inefficiencies can only be resolved with exclusion contracts or buy-out contracts. If launch inefficiencies are of concern (high $\alpha_{L}$ ), this is best addressed through buy-out contracts. Otherwise, exclusion contracts are preferred as they generate the strongest research incentive.

\subsection{Full Contracts: Upfront and Milestone Payments and Royalties}

Our analysis so far has focused on contracts with either milestone payments or royalties. Nat-

urally, designing contracts with both elements may lead to higher contract values. However, this is not always the case as shown by Proposition 2 .

\section{Proposition 2}

1. Milestone contracts without a royalty rate are optimal (a) if the milestone contract with innovator launch control achieves social optimum or (b) if an exclusion contract is optimal.

2. It is optimal to set $l=0$ when $\gamma=-1$. 


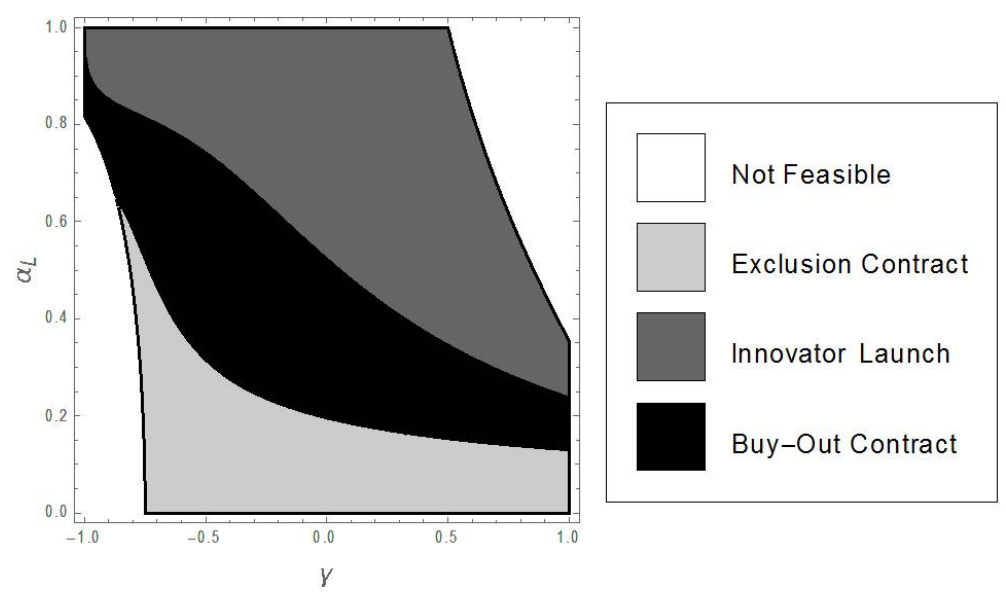

Figure 3: Optimal contract structure: Launch control and buy-out option $\left(\alpha_{H}=1, k_{M}=1.5, k_{I}=4, p_{L}=0.25\right)$

In cases not covered by Proposition 2 it may be beneficial to include all payment terms in the contract. We resort to numerical analyses for a comparison of the different contracting choices.

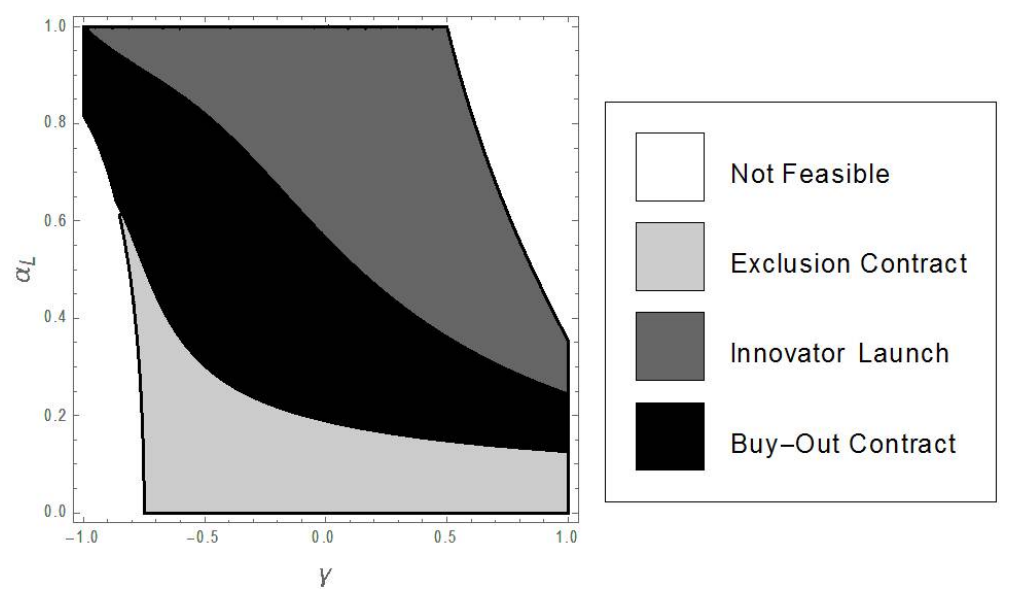

Figure 4: Optimal contract structure with full contracts $\left(\alpha_{H}=1, k_{M}=1.5, k_{I}=4, p_{L}=0.25\right)$

It is clear from Figure 4 that the results for full contracts display a very similar pattern of contract structure choices as the milestone-only or royalty-only contracts. The actual values taken on by the payment terms, however, are influenced both by the optimal contract structure and the choice to allow full contracts. Our results confirm the intuition that the optimal royalty rate is much higher when a buy-out contract is optimal than for other contract structures. 


\section{Renegotiation}

The allocation and exercise of control rights and options is not the only way to react to new information: as uncertainties are resolved over time, the two parties may choose to renegotiate the initial contract. While Plambeck and Taylor (2007) suggest that renegotiation is often excluded by papers in operations management on the grounds that it is prohibitively costly, the authors show that renegotiation can be optimal. So far in our paper, we have implicitly assumed that renegotiation is prohibitively costly. In this section, we explore the cases when renegotiation is costless or moderately costly. We focus most of our efforts on the less explored intermediate cost case where renegotiation is neither prohibitively costly nor costless.

If renegotiation is costless, Hart and Moore (1988) and Nöldeke and Schmidt (1995) show that renegotiation can be beneficial and even help achieve the first-best outcome under certain conditions. In our model, renegotiation could be instigated once new information becomes available, i.e., the $\mathrm{R} \& \mathrm{D}$ outcome is revealed, but before the contract terms are executed and the marketer's effort level is decided. Renegotiation occurs whenever there are efficiency gains to be realized because the initial contract distorts effort or distorts launch decisions and a new contract can be written that leaves both parties better off. In line with Hart and Moore (1988), the innovator will invest the optimal R\&D effort level if she is the recipient of all the ex-post surplus. This can be achieved by writing an exclusion contract with a milestone payment $l=\alpha_{H}^{2} /\left(2 k_{M}\right)$ that the innovator will subsequently offer to negotiate down to $l=\alpha_{L}^{2} /\left(2 k_{M}\right)$ whenever the market-potential is low. This gives the innovator the full surplus regardless of the market-potential without distorting the marketer's effort level.

Adding renegotiation costs clearly reduces the attractiveness of renegotiation and if the cost of renegotiation exceeds the benefits achieved by renegotiation, no Pareto-improving renegotiation can take place. Various papers acknowledge that renegotiation is neither costless nor prohibitively costly (e.g. Garleanu and Zwiebel 2009). We model the cost of renegotiation as a fixed cost, $F>0$, that is entirely borne by the principal, the innovator, at the time of renegotiation and study the previously discussed contract types in turn.

Milestone Contracts As milestone contracts do not lead to distortion of marketing effort, the only benefit from renegotiation is when the launch decision is distorted and we can focus exclusively on exclusion contracts. The innovator may find it beneficial to renegotiate an exclusion contract in case of a low market-potential outcome. Inserting the value of the future renegotiation into the innovator's initial problem formulation, this leads to the 
following optimization problem:

$$
\max _{s, l} e l+\left(p_{L}+\gamma e\right)\left(\frac{\alpha_{L}^{2}}{2 k_{M}}-F\right)-\frac{e^{2}}{2}+s
$$

Proposition 3 If $F \leq \frac{\alpha_{L}^{2}}{2}\left(\frac{1}{k_{M}}-\frac{1}{k_{I}}\right)$, the exclusion contract will be renegotiated in the low market-potential outcome. The optimal contract terms are $\{s, l\}=\left\{0, \frac{\alpha_{H}^{2}}{2 k_{M}}\right\}$, and lead to the following optimal R\&D effort and contract value:

$$
e^{*}=\frac{\alpha_{H}^{2}+\gamma \alpha_{L}^{2}}{2 k_{M}}-\gamma F, V^{*}=\frac{\left(\alpha_{H}^{2}+\alpha_{L}^{2} \gamma\right)^{2}}{2 k_{M}^{2}}+\frac{\left(2\left(\alpha_{L}^{2} p_{L}-F \gamma\left(\alpha_{H}^{2}+\alpha_{L}^{2} \gamma\right)\right)\right.}{k_{M}}-F\left(2 p_{L}-\gamma^{2} F\right)
$$

Buy-Out Option Contract In an option contract, efficiency gains can be achieved by eliminating the effort-distorting royalty rate in the low market-potential outcome. The innovator's optimization problem becomes:

$$
\max _{s, r} e \frac{r(2-r) \alpha_{H}^{2}}{2 k_{M}}+\left(p_{L}+\gamma e\right)\left(\frac{r(2-r) \alpha_{L}^{2}}{2 k_{M}}-F\right)-\frac{e^{2}}{2}+s
$$

Renegotiation is only ex-post rational when the renegotiation cost is $F \leq \frac{\alpha_{L}^{2} r^{2}}{2 k_{M}}$. As we cannot write a closed form expression for the optimal royalty rate in the absence of renegotiation, we focus instead on the intuition behind the optimal buy-out contract in the presence of costly renegotiation. Assume that without renegotiation, the optimal royalty rate is $r_{1}$ (as calculated in section 4.2.1. Two cases may occur: $F \leq \frac{\alpha_{L}^{2} r_{1}^{2}}{2 k_{M}}$ or $F>\frac{\alpha_{L}^{2} r_{1}^{2}}{2 k_{M}}$. In the first case, renegotiation is profitable, whereas in the second it is not. Let us take the first case, in which renegotiation occurs. If the buy-out contract induces renegotiation, then the minimum amount of distortion of the $R \& D$ effort is trivially achieved when the royalty rate is set to $r=1$. In the high market-potential outcome, the marketer exercises his buy-out option priced at $B=\alpha_{H}^{2} /\left(2 k_{M}\right)$, and in the low market-potential outcome, renegotiation achieves a net payoff to the innovator of $\alpha_{L}^{2} /\left(2 k_{M}\right)-F$. Note that these are exactly the same payoffs as in the milestone contract with marketer launch and renegotiation. In the second case, renegotiation is not ex-post optimal, and the optimal royalty rate is $r^{*}=r_{1}$. The contract value is the same as in the buy-out contract without renegotiation studied in section 4.2.1. A comparison of the two contract values then allows us to determine whether the buy-out contract should be structured so as to achieve renegotiation by setting $r=1$ or not by setting $r=r_{1}$.

We present a comparison of all contracts covered and costly renegotiation in Figure 5. Remember that our previous analysis shows that buy-out contracts with renegotiation are equivalent to exclusion contracts with renegotiation. Renegotiation is only optimal when 
the various efficiency losses of the upfront contracts exceed the cost of renegotiation. As marketing and research inefficiencies are highest for incremental products with low marketpotential variability, renegotiation is optimal in the top left quadrant of the figure. Whenever renegotiation is too costly, the tradeoffs between other regions of the figure are the same as in Figure 3 .

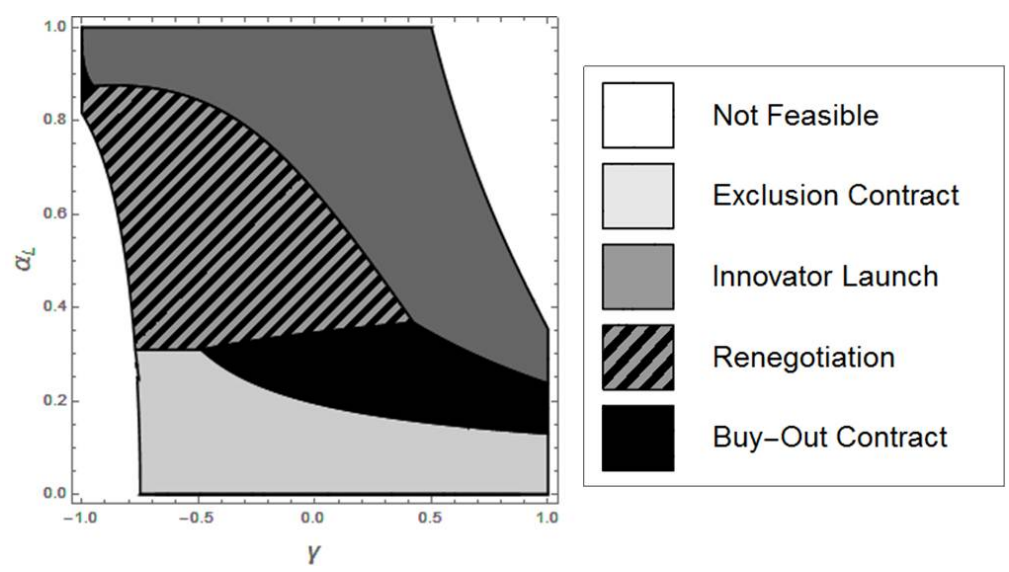

Figure 5: Optimal Contracts with Costly Renegotiation $\left(\alpha_{H}=1, k_{M}=1.5, k_{I}=4, p_{L}=0.25, F=0.02\right)$

\section{Delayed Contracting}

While there may be forces extraneous to our paper such as risk aversion or cash constraints that push the innovator to contract early in the R\&D cycle, in the absence of such motives for upfront contracting, the innovator may choose to bide her time and delay contracting with the marketer until his contribution is needed. At this time, the R\&D effort has been made and its outcome realized. If contracting is costless and market-potential is observable, the first-best solution can be trivially obtained. The innovator simply sells the product to the marketer at its market value, yielding the right incentives for both the innovator and the marketer. We now relax these cost and observability conditions in turn.

\subsection{Costly Contracting}

While contracting is often assumed to be costless, we have argued before that this is unlikely to be the case: legal fees or delays in development reduce the value of the R\&D project, thus affecting the contract value that can be created. We introduce a fixed cost for upfront and delayed contracting, denoted by $F_{1}$ and $F_{2}$ respectively. To avoid the trivial scenario in which contracting is never optimal, even after the $\mathrm{R} \& \mathrm{D}$ outcome is revealed, we limit $F_{2}<\alpha_{H}^{2} /\left(2 k_{M}\right)$. 
Adding a fixed cost to the innovator's optimization problem for upfront contracting does not change her optimal contract choice. The cost $F_{1}$ can be deducted from the optimal contract value determined as per our prior analysis. The benefits and drawbacks of the different contractual choices still apply. However, we also note that upfront contracting costs are incurred regardless of whether the project turns out to be successful or not. When the innovator delays contracting, she incurs a fixed cost $F_{2}$, and, given that market-potential is observable, the project is sold for a fixed price equivalent to its market value. The innovator's optimization problem becomes:

$$
\max _{e} e\left(\frac{\alpha_{H}^{2}}{2 k_{M}}-F_{2}\right)+\left(p_{L}+\gamma e\right) \max \left\{\frac{\alpha_{L}^{2}}{2 k_{M}}-F_{2}, \frac{\alpha_{L}^{2}}{2 k_{I}}\right\}-\frac{e^{2}}{2}
$$

From the point of view of efficiency, we observe two opposing forces. On the upside, delayed contracting avoids royalties thereby ensuring optimal marketing effort from the marketer. On the downside, the innovator's R\&D effort is distorted due to the future contracting $\operatorname{cost} F_{2}$ and an inefficient launch decision may be taken if the cost of contracting is too high to justify selling to the marketer in the low market-potential outcome $\left(F_{2}>\alpha_{L}^{2} / 2\left(1 / k_{M}-1 / k_{I}\right)\right)$ and the innovator takes the project forward herself. The optimal R\&D effort and contract value are summarized below.

Proposition 4 If the innovator chooses to delay contracting, the optimal R\&D effort and contract value are given by the expressions below whenever $F_{2} \leq \alpha_{L}^{2} / 2\left(1 / k_{M}-1 / k_{I}\right)$.

$e^{*}=\frac{\alpha_{H}^{2}+\alpha_{L}^{2} \gamma}{2 k_{M}}-(1+\gamma) F_{2}, V^{*}=\frac{\left(\alpha_{H}^{2}+\alpha_{L}^{2} \gamma\right)^{2}-\left(\alpha_{H}^{2}+\alpha_{L}^{2}\right) F_{2}(1+\gamma)+\alpha_{L}^{2} p_{L}}{2 k_{M}}+\frac{F_{2}\left(F_{2}(1+\gamma)^{2}-2 p_{L}\right)}{2}$

If $F_{2}>\alpha_{L}^{2} / 2\left(1 / k_{M}-1 / k_{I}\right)$, the optimal R\&D effort and contract value are:

$e^{*}=\frac{\alpha_{H}^{2} k_{I}+\alpha_{L}^{2} \gamma k_{M}}{2 k_{I} k_{M}}-F_{2}, V^{*}=\frac{\left(\alpha_{H}^{2} k_{I}+\alpha_{L}^{2} \gamma k_{M}\right)^{2}}{8 k_{I}^{2} k_{M}^{2}}-\frac{\left(\alpha_{H}^{2} k_{I}+\alpha_{L}^{2} \gamma k_{M}\right) F_{2}+\alpha_{L}^{2} \gamma p_{L} k_{M}+k_{I} k_{M} F_{2}^{2}}{2 k_{I} k_{M}}$

The choice between upfront or delayed contracting needs to balance the benefits and costs of the different timing options. We can see from Figure 6 that either delayed contracting or one of the previously discussed milestone or option contracts may be optimal. Similar to the discussion about renegotiation, delayed contracting is optimal whenever the research and marketing inefficiencies of upfront contracting are high, i.e., in the top left quadrant. Intuitively, the region where delayed contracting is optimal will expand or shrink depending on the relative cost of upfront vs. delayed contracting. 


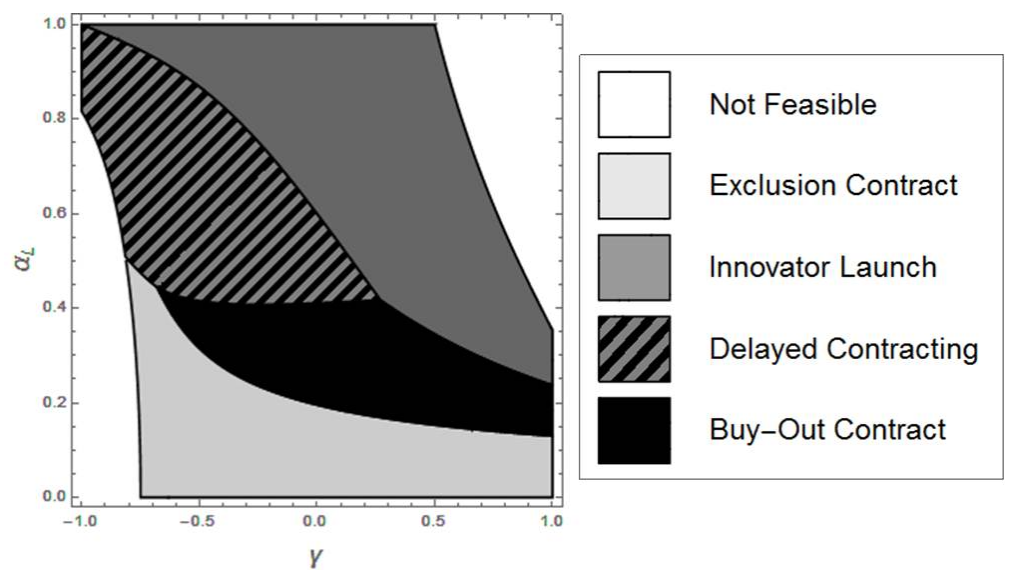

Figure 6: Optimal Contracts with Delayed Contracting $\left(\alpha_{H}=1, k_{M}=1.5, k_{I}=4, p_{L}=0.25, F_{1}=0.005, F_{2}=0.02\right)$

\subsection{Unobservable market-potential}

As previously discussed, delayed contracting can achieve first-best if contracting is costless and market-potential is observable. Having analyzed the impact of relaxing the first of these conditions, we now relax the observability assumption.

The innovator observes failure versus success at the end of the research phase but marketpotential is only observable to the marketer. This leads to a principal-agent problem at $t=4$ where the principal (innovator) knows the probabilities of the possible outcomes, while the agent (marketer) knows the outcome. We adopt the terminology of adverse selection problems and label the marketer facing a high (low) market-potential outcome as the H-type (L-type). Our setup is a variation from the typical principal-agent model where probabilities for types are exogenous; they are endogenous in our case. The innovator solves the following optimization problem and selects contract parameters, as a function of her effort. The incentive compatibility constraints (ICH and ICL) ensure that each type of marketer prefers the contract intended for his own type while the individual rationality constraints (IRH and IRL) ensure that both types participate. 


$$
\begin{array}{ccc}
\max _{0 \leq l_{i}, 0 \leq r_{i} \leq 1} \Omega\left(e, l_{L}, l_{H}, r_{L}, r_{H}\right)=\pi_{L}(e)\left(l_{L}+\frac{r_{L}\left(1-r_{L}\right) \alpha_{L}^{2}}{k_{M}}\right)+\pi_{H}(e)\left(l_{H}+\frac{r_{H}\left(1-r_{H}\right) \alpha_{H}^{2}}{k_{M}}\right) \\
\text { s.t. } & \frac{\left(1-r_{H}\right)^{2} \alpha_{H}^{2}}{2 k_{M}}-l_{H} \geq \frac{\left(1-r_{L}\right)^{2} \alpha_{H}^{2}}{2 k_{M}}-l_{L} \quad \text { (ICH) } \\
& \frac{\left(1-r_{L}\right)^{2} \alpha_{L}^{2}}{2 k_{M}}-l_{L} \geq \frac{\left(1-r_{H}\right)^{2} \alpha_{L}^{2}}{2 k_{M}}-l_{H} \\
& \frac{\left(1-r_{H}\right)^{2} \alpha_{H}^{2}}{2 k_{M}}-l_{H} \geq 0 & \text { (ICL) } \\
& \frac{\left(1-r_{L}\right)^{2} \alpha_{L}^{2}}{2 k_{M}}-l_{L} \geq 0 & \text { (IRH) }
\end{array}
$$

Proposition 5 It is optimal for the innovator to offer a menu of two contracts. One intended for the H-type marketer with

$$
l_{H}^{*}=\frac{\left(2 r_{L}^{*}-r_{L}^{* 2}\right) \alpha_{H}^{2}+\left(1-r_{L}^{*}\right)^{2} \alpha_{L}^{2}}{2 k_{M}}, r_{H}^{*}=0
$$

and another intended for the L-type marketer with

$$
l_{L}^{*}=\frac{\left(1-r_{L}^{*}\right)^{2} \alpha_{L}^{2}}{2 k_{M}}, r_{L}^{*}=\frac{\pi_{H}(e)\left(\alpha_{H}^{2}-\alpha_{L}^{2}\right)}{\pi_{H}(e)\left(\alpha_{H}^{2}-\alpha_{L}^{2}\right)+\pi_{L}(e) \alpha_{L}^{2}} .
$$

The L-type marketer receives a contract with milestones and royalties that transfers all value created from the partnership to the innovator. Intuitively, royalties cause a larger distortion for the H-type marketer who enjoys a more valuable product. The innovator designs a contract with higher milestones $l_{H}>l_{L}$ for the H-type marketer and zero royalties. While the lower milestone is attractive to the H-type marketer, the value destroyed by royalties is much higher for the H-type marketer than the L-type, thereby discouraging him from choosing the contract intended for the L-type. This allows for separation of the two types. The H-type marketer enjoys an information advantage and makes a positive profit.

Theorem 6 Under unobservable market-potential, an upfront full contract with a buy-out option outperforms both delayed contracting and an upfront buy-out option without an upfront payment.

While the information advantage of the marketer can be partially addressed by a menu of contracts when delayed contracting leads to unobservable market-potential, the marketer would still retain some information rents. Theorem 6 shows that a full contract, which uses all three payment terms $(s, l, r)$, signed upfront when neither party has superior information allows the innovator not to give information rents. To see this, first note that the menu of contracts described in Proposition 5 can be replicated by a single buy-out contract with 
$l=l_{L}, r=r_{L}$ and $B=l_{H}-l_{L}=\frac{\left(2 r-r^{2}\right) \alpha_{H}^{2}}{2 k_{M}}$, the payment of which relieves the marketer of the obligation to pay royalties. Second, the positive rents enjoyed by the H-type can be extracted by an upfront signature fee $s>0$, at time $\mathrm{t}=1$, that does not interfere with incentive compatibility or product launch decisions, at time $t=4$ where the signature fee is a sunk cost for the marketer.

\section{Robustness}

Given the centrality of the parameter $\gamma$ to our model, we test the robustness of our results to an alternate model specification in which the innovator can invest in two different types of research efforts: research effort $e_{P}$ enhances the probability of technical success whereas research effort $e_{Q}$ increases the probability of a high market-potential given technical success. We list the necessary notation changes in Table 3 . All variables not mentioned in the table are as per the original model. Similar to our original model, we make the following assumptions: the probabilities are linear in research effort, $\pi\left(e_{P}\right)=p+e_{P}$ for the probability of technical success and $\kappa\left(e_{Q}\right)=q+e_{Q}$ for the probability of high market-potential given technical success; and the cost of both research efforts is quadratic in effort, i.e. $c_{P} e_{P}^{2} / 2$ and $c_{Q} e_{Q}^{2} / 2$, respectively.

Decisions

Parameters

\begin{tabular}{llll}
\hline$e_{P}$ & Research effort to increase & $p$ & Base probability of technical success \\
& probability of technical success & $c_{P}$ Cost factor of research effort $e_{P}$ \\
$e_{Q}$ & Research effort to increase & $q$ & Base probability of high market-potential \\
& probability of high market-potential & $c_{Q}$ Cost factor of research effort $e_{Q}$
\end{tabular}

Table 3: Notation for Model with Two Research Efforts

When marketing effort is undertaken by the marketer, the project value is:

$V=\left(p+e_{P}\right)\left(\left(q+e_{Q}\right)\left(\alpha_{H} \mu_{H M}-\frac{k_{M} \mu_{H M}^{2}}{2}\right)+\left(1-q-e_{Q}\right)\left(\alpha_{L} \mu_{L M}-\frac{k_{M} \mu_{L M}^{2}}{2}\right)\right)-\frac{c_{P} e_{P}^{2}}{2}-\frac{c_{Q} e_{Q}^{2}}{2}$

A project for which $c_{P}$ is high compared to $c_{Q}$ corresponds to a incremental project: improvements aiming to reach higher market-potential are easier to make, but the probability of technical success is difficult to increase. This corresponds to $\gamma<0$ in the original model. Conversely, $c_{P}$ could be relatively low compared to $c_{Q}$ : this maps to novel projects, where it is easier to work on ensuring that the project will be technically successful rather than working toward the high market-potential outcome. By varying the cost of increasing the probability of a high market-potential outcome, $c_{Q}$, while keeping the cost of investing in enhancing the 
technical probability of success, $c_{P}$, constant, we span the spectrum of R\&D process types from incremental to novel. We analyze the same contract structures and payment terms for upfront contracting as for our previous model and plot the results in Figure 7.

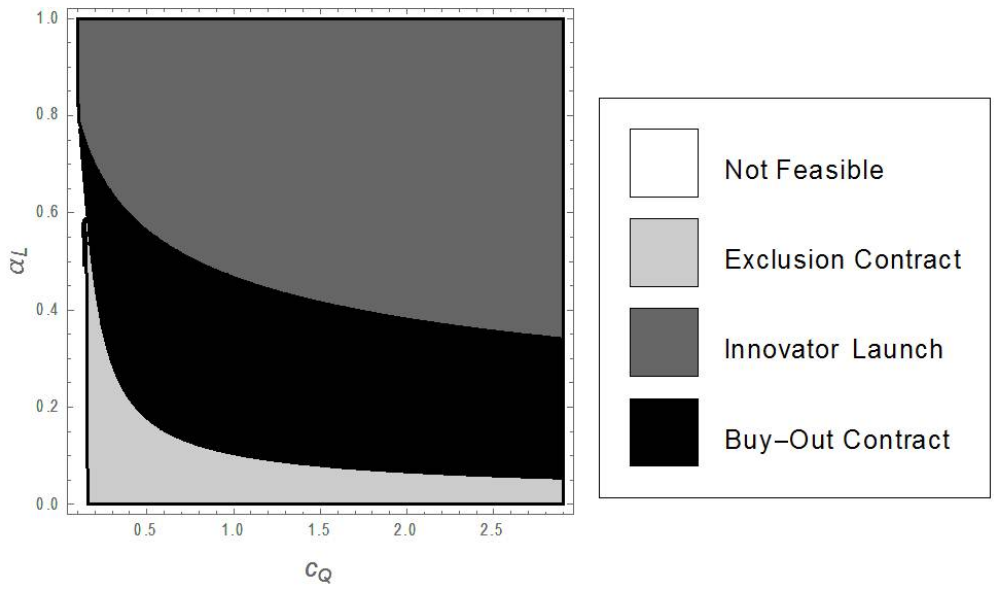

Figure 7: Optimality of Upfront Contracts

$$
\left(\alpha_{H}=1, k_{I}=1.5, c_{P}=1.5, k_{M}=3, p=0.25, q=0\right)
$$

Comparing Figures 3 and 7 , we observe that our qualitative insights are robust to the model specifications as the same contract types are optimal for similar combinations of project parameters. An exclusion contract is optimal for large differences between low and high market-potential outcomes. As an exclusion contract provides strong incentives for reaching the high market-potential outcome, it is less likely to be the optimal contract choice when the cost of increasing the probability of a high market-potential outcome is high. In contrast, the milestone contract with innovator launch control provides a strong incentive for enhancing the technical probability of success. Hence, it is more likely to be optimal when the cost of increasing the probability of a high market-potential outcome is high or when there is low market-potential variability, as this makes the market-potential outcome less relevant. The buy-out contract is optimal for the remaining combinations of project parameters, i.e., when the contract should induce some market-potential enhancing effort, but the low market-potential outcome is still valuable enough that it should be marketed by the more efficient party, the marketer.

\section{Conclusion}

R\&D projects and partnerships are difficult to manage due to inherent uncertainties with respect to both successful product launch and market value after commercialization. Two parties may bring different capabilities to the partnership, which improves the value that can be attained. At the same time, the inclusion of a second party raises incentive alignment 
concerns and leads to various inefficiencies. Any contract that governs the relationship between the two parties should be designed to obtain the appropriate amount of effort from both and align incentives.

In this paper, we study how control rights, options and timing can be used, in conjunction with various payment terms, to address the inefficiencies that may occur when innovators and marketers form partnerships to develop and market new products. We find that the key factors driving the optimal structure and timing decision depends on the type of $R \& D$ process and the variability in market potential outcomes. Our analysis leads to several managerial implications.

Much of the licensing literature has focused on the optimization of various payment terms within a given contract structure. We find that it is important for parties contemplating an alliance to consider contracting options holistically because clauses allocating launch control or options to either party and contract timing all have an impact on the effectiveness of different payment terms. Our analysis shows that the joint optimization of contract structure and payment terms leads to various contract structures being optimal under different R\&D project characteristics. Furthermore, our investigation into the timing of the contracting decision in the presence of negotiation costs highlight that either upfront or delayed contracting may be optimal. This shows that optimizing only payment terms is suboptimal and the simultaneous optimization of contract structure, timing, and payment terms is key for improving the value attained from $R \& D$ partnerships.

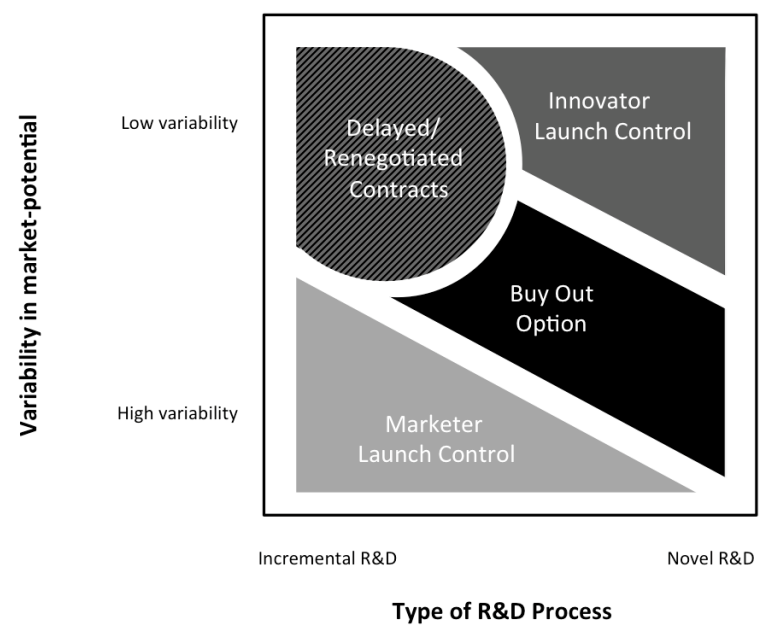

Figure 8: Mapping of Contract Choices

We identify two forces that drive contracting choices: (a) whether the innovation process is novel or incremental and (b) variability in market-potential outcomes. In Figure 8, we provide a map for managers entering R\&D partnerships, which guides contract choice based 
on these factors. The mapping exploits the interaction of the contract terms with those two factors to manage three distinct efficiency losses resulting from suboptimal R\&D effort, marketing effort, or launch allocation. The R\&D process type affects the ability of the different payment terms to achieve efficient $R \& D$ effort, while variability in market-potential affects the magnitude of the two other efficiency losses. Thus, incremental products with high variability in market-potential outcomes are best assigned to exclusion contracts, which limit distortion to marketing and R\&D efforts and suffer a comparatively mild loss due to innovator launch in the low market-potential outcome. For novel innovations with low market-potential variability, innovator launch control does not distort the marketing effort and achieves marketer launch with only a slight distortion of R\&D effort. Novel innovations with high market-potential variability require payment terms that are more responsive to the market outcome, thus favoring buy-out contracts or exclusion contracts. Finally, incremental products with low variability may be best served by delaying contracting or by renegotiating an upfront contract to avoid costly marketing effort or launch allocation distortions.

This study further allows us to highlight an interesting result: the innovator may actually be better off curtailing her rights and granting them to the marketer. First, in an exclusion contract, the launch control right is transferred to the marketer to create a credible commitment to not claiming a reward (milestone) under the low market-potential outcome, creating an incentive to exert higher R\&D effort and achieve the high market-potential outcome. Second, in a buy-out contract, the innovator allows the marketer to buy her out whenever the project achieves high market-potential. Similarly, this buy-out payment creates the necessary incentives for the innovator to exert more effort to reach the high market-potential outcome. While the benefits of holding an option are well understood, this shows that giving away an option can also be valuable.

Our work and its insights are timely as evidenced by the growing importance of R\&D partnerships (Roijakkers and Hagedoorn 2006) and the common practice of including control rights and options in R\&D contracts, as illustrated by the industry examples of Section 1 . By modeling a combination of contract payments, the allocation of control rights and options, and timing decisions, our work yields valuable insights into the timing and structure of R\&D contracts and opens avenues for further empirical work.

\section{Appendix}

\section{Proof of Theorem 1 .}

The objective function is concave in $l$ and the feasible space is convex. The solution described in Theorem 1 follows directly from the Karush-Kuhn-Tucker (KKT) conditions to 
the problem.

Formulating the Lagrangian of the optimization problem described by Equations (5)-(8) and substituting the research and marketing effort levels gives us:

$$
\frac{\alpha_{H}^{2}\left(1+\lambda_{1}\right)(l+\gamma l)+\alpha_{L}^{2}\left(1+\lambda_{1}\right)\left(\gamma(1+\gamma) l+p_{L}\right)-k_{M} l\left((1+\gamma)^{2}\left(1+2 \lambda_{1}\right) l+2\left(\lambda_{1} p_{L}-\lambda_{2}\right)\right)}{2 k_{M}}
$$

Case 1. $\lambda_{1}=\lambda_{2}=0$. This case occurs when $p_{L}=0$. The optimal contract is $(s, l)=$ $\left(0, \frac{\alpha_{H}^{2}+\alpha_{L}^{2} \gamma}{2(1+\gamma) k_{M}}\right)$. This contract achieves the socially optimal value.

Case 2. $\lambda_{1}=0, \lambda_{2}>0$. This implies a contract with upfront payment $s$ only $(l=0)$. Solving the system, we find that $\lambda_{2}=-\frac{(1+\gamma)\left(\alpha_{H}^{2}+\alpha_{L}^{2} \gamma\right)}{2 k_{M}}$. This is negative except when $\gamma=-1$. Impossible.

Case 3. $\lambda_{1}>0, \lambda_{2}=0$. This case occurs when $p_{L}>0$ and implies a contract with a milestone payment $l$ only $(s=0)$. This yields the following optimal contract terms $(s, l)=\left(0, \frac{\left(\alpha_{H}^{2}+\alpha_{L}^{2} \gamma\right)(1+\gamma)-2 k_{M} p_{L}-\sqrt{8(1+\gamma)^{2} k_{M} \alpha_{L}^{2} p_{L}+\left((1+\gamma)\left(\alpha_{H}^{2}+\alpha_{L}^{2} \gamma\right)-2 k_{M} p_{L}\right)^{2}}}{4(1+\gamma)^{2} k_{M}}\right)$. This contract does not achieve the socially optimal value.

Case 4. $\lambda_{1}>0, \lambda_{2}>0$. This implies that $(s, l)=(0,0)$. No contract is written.

Proof of Theorem 2 .

For both milestone contracts with marketer launch control, the milestone payment and the corresponding contract value are trivial to determine.

If both outcomes are to be launched by the marketer, the milestone payment will be $l=\frac{\alpha_{L}^{2}}{2 k_{M}}$ and the contract value is $\Omega^{1}=\frac{4 k_{M} \alpha_{L}^{2} p_{L}+2 \alpha_{L}^{2} \alpha_{H}^{2}(1+\gamma)-\alpha_{L}^{4}\left(1-\gamma^{2}\right)}{8 k_{M}^{2}}$. An exclusion contract will have a milestone payment $l=\frac{\alpha_{H}^{2}}{2 k_{M}}$ and contract value $\Omega^{2}=p_{L} \frac{\alpha_{L}^{2}}{2 k_{I}}+\frac{\left(\alpha_{H}^{2} /\left(2 k_{M}\right)+\gamma \alpha_{L}^{2} /\left(2 k_{I}\right)\right)^{2}}{2}$.

Let us compare $\Omega^{1}$ and $\Omega^{2}$ :

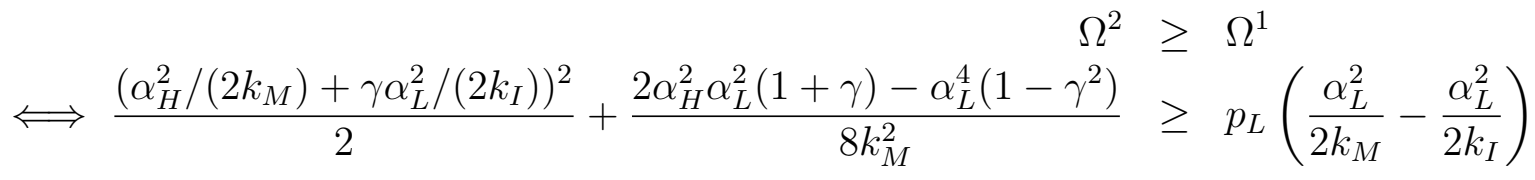

The RHS of this expression is constant in $\gamma$ whereas the LHS of this expression is decreasing in $\gamma$. We can find an interior solution $\hat{\gamma} \in[-1,1]$ such that for all $\gamma \geq \hat{\gamma}, \Omega^{1} \geq \Omega^{2}$ except when $(1) \frac{\left(\alpha_{H}^{2} /\left(2 k_{M}\right)-\alpha_{L}^{2} /\left(2 k_{I}\right)\right)^{2}}{2} \leq p_{L}\left(\frac{\alpha_{L}^{2}}{2 k_{M}}-\frac{\alpha_{L}^{2}}{2 k_{I}}\right)$, in which case an exclusion contract is never optimal or $(2) \frac{\left(\alpha_{H}^{2} /\left(2 k_{M}\right)+\alpha_{L}^{2} /\left(2 k_{I}\right)\right)^{2}}{2}+\frac{\alpha_{H}^{2} \alpha_{L}^{2}}{2 k_{M}^{2}} \geq p_{L}\left(\frac{\alpha_{L}^{2}}{2 k_{M}}-\frac{\alpha_{L}^{2}}{2 k_{I}}\right)$, in which case an exclusion contract is always optimal.

\section{Proof of Theorem 3}

If $k_{I}>k_{M}$, the milestone contract with innovator launch control (contract value $\Omega^{3}$ ) dominates the milestone contract with marketer launch control when both outcomes are launched. Hence, for all $\gamma \geq \hat{\gamma}, \Omega^{3} \geq \Omega^{1} \geq \Omega^{2}$. Thus a $\tilde{\gamma}$ that satisfies that for all $\gamma \geq \tilde{\gamma}$ we have $\Omega^{3} \geq \Omega^{2}$ and for all $\gamma<\tilde{\gamma}$ we have $\Omega^{3}<\Omega^{2}$ would have to be less than or equal to $\hat{\gamma}$. 
If there exists an interior $\hat{\gamma} \in]-1,1]$, then at $\gamma=-1$, we have:

$$
\Omega^{2}=p_{L} \frac{\alpha_{L}^{2}}{2 k_{I}}+\frac{\left(\alpha_{H}^{2} /\left(2 k_{M}\right)-\alpha_{L}^{2} /\left(2 k_{I}\right)\right)^{2}}{2} \geq \Omega^{1}=p_{L} \frac{\alpha_{L}^{2}}{2 k_{M}}=\Omega^{3},
$$

and the region over which $\Omega^{2} \geq \Omega^{3}$ is non-empty.

\section{Proof of Proposition 1}

In the optimization problem given by equations (15)-(18) we use the constraints to substitute three variables as a function of $r$ as follows: (a) the marketing effort $\mu_{i M}$ (Equation (16)); (b) the research effort $e$ (Equation (17)) and (c) the upfront payment $s$ (Equation (18)).

Note that the last substitution is legitimate because the resulting upfront payment will always be non-negative: any royalty rate $r \leq 1$ leaves the marketer with a non-negative profit in both market-potential outcomes and the upfront payment is the expected value of the marketer's profit after royalty payments leaving the marketer with zero surplus.

This gives us an unconstrained optimization problem with one decision variable, $r$ :

$$
\Omega^{1}=\frac{(1-r)\left(\left(\alpha_{H}^{2}+\alpha_{L}^{2} \gamma\right)^{2}(1-r) r+k_{M}(1+r) \alpha_{L}^{2} p_{L}\right)}{2 k_{M}^{2}}
$$

Then, the solution to the first-order condition of that problem is:

$$
\begin{aligned}
r^{*} & =\frac{2\left(\alpha_{H}^{2}+\alpha_{L}^{2} \gamma\right)^{2}+k_{M} \alpha_{L}^{2} p_{L}-\sqrt{-3\left(\alpha_{H}^{2}+\alpha_{L}^{2} \gamma\right)^{4}+\left(2\left(\alpha_{H}^{2}+\alpha_{L}^{2} \gamma\right)^{2}+k_{M} \alpha_{L}^{2} p_{L}\right)^{2}}}{3\left(\alpha_{H}^{2}+\alpha_{L}^{2} \gamma\right)^{2}} \\
& =\frac{2}{3}-\frac{\sqrt{\left(\left(\alpha_{H}^{2}+\alpha_{L}^{2} \gamma\right)^{2}+k_{M} \alpha_{L}^{2} p_{L}\right)^{2}+2 k_{M} \alpha_{L}^{2} p_{L}\left(\alpha_{H}^{2}+\alpha_{L}^{2} \gamma\right)^{2}}-k_{M} \alpha_{L}^{2} p_{L}}{3\left(\alpha_{H}^{2}+\alpha_{L}^{2} \gamma\right)^{2}}
\end{aligned}
$$

It can be verified that on the interval $\left[r^{*}, 1\right]$ the first-order condition is non-positive. The second-order condition is negative at $r^{*}$.

Substituting $p_{L}=0$ gives $r^{*}=1 / 3$. The derivative of $r^{*}$ w.r.t. $p_{L}$ is negative and the optimal royalty rate is decreasing in $p_{L}$. The second equation line shows that $r^{*}$ never exceeds $1 / 3$.

\section{Proof of Theorem 4}

A buy-out contract with royalty rate $r=100 \%$ and buy-out price $B=\frac{\alpha_{H}^{2}}{2 k_{M}}$ is equivalent to an exclusion contract, and a buy-out contract with royalty rate set to the optimal royalty rate of the royalty contract with a buy-out price in excess of $\frac{r(2-r) \alpha_{H}^{2}}{2}$ is equivalent to the optimal royalty contract. Thus, the optimal buy-out contract will perform at least as well as the best of both.

Take $\Omega^{1}$ and $\Omega^{2}$ as the innovator's objective function without and with buy-out option. (We substitute the marketing effort, research effort and upfront payment as a function of $r$ in 
$\Omega^{2}$ as described in the proof of Proposition 1 thus obtaining an unconstrained optimization problem.)

$$
\frac{\partial \Omega^{2}}{\partial r}-\frac{\partial \Omega^{1}}{\partial r}=\frac{\alpha_{H}^{2} r\left(\alpha_{H}^{2}+\alpha_{L}^{2} \gamma-\left(\alpha_{H}^{2}+2 \alpha_{L}^{2} \gamma\right) r^{2}\right)}{2 k_{M}^{2}}
$$

The difference is positive for $r \leq \sqrt{\frac{\alpha_{H}^{2}+\alpha_{L}^{2} \gamma}{\alpha_{H}^{2}+2 \alpha_{L}^{2} \gamma}}$. Write the optimal royalty rate without buy-out option $r^{1}$. By Proposition 1, we know that $r^{1} \leq 1 / 3$ and at $r^{1}, \frac{\partial \Omega^{1}}{\partial r}=0$. We also show that:

$$
\begin{aligned}
\frac{1}{3} & \leq \sqrt{\frac{\alpha_{H}^{2}+\alpha_{L}^{2} \gamma}{\alpha_{H}^{2}+2 \alpha_{L}^{2} \gamma}} \\
\alpha_{H}^{2}+2 \alpha_{L}^{2} \gamma & \leq 9\left(\alpha_{H}^{2}+\alpha_{L}^{2} \gamma\right) \\
0 & \leq 8 \alpha_{H}^{2}+7 \alpha_{L}^{2} \gamma
\end{aligned}
$$

Thus, at $r^{1}, \frac{\partial \Omega^{2}}{\partial r}-0=\frac{\alpha_{H}^{2} r\left(\alpha_{H}^{2}+\alpha_{L}^{2} \gamma-\left(\alpha_{H}^{2}+2 \alpha_{L}^{2} \gamma\right) r^{2}\right)}{2 k_{M}^{2}} \geq 0$. As the first order derivative of $\Omega^{2}$ is positive at $r^{1}$, the optimal royalty rate with buy-out option, $r^{2}$, is larger than $r^{1}$.

\section{Proof of Theorem 5}

The innovator can set the buy-out option strike price to either $B_{1}=\frac{\alpha_{H}^{2} r(2-r)}{2 k_{M}}$ and have the marketer exercise the option in only the $\alpha_{H}$ scenario or set it to $B_{1}=\frac{\alpha_{L}^{2} r(2-r)}{2 k_{M}}$ and have him exercise the option in both the $\alpha_{H}$ and $\alpha_{L}$ scenarios. Note that when the innovator's strike price, $B_{2}>\frac{\alpha_{L}^{2}}{2 k_{I}}-\frac{r(1-r) \alpha_{L}^{2}}{k_{M}}$, the innovator never exercises her option and the two-way option contract is equivalent to the buy-out contract characterized by Theorem 4 . We therefore investigate the case where the innovator would exercise her option which means that the strike price has to satisfy $0 \leq B_{2} \leq \frac{\alpha_{L}^{2}}{2 k_{I}}-\frac{r(1-r) \alpha_{L}^{2}}{k_{M}}$. As she prefers a lower strike price, we will set the innovator's strike price to $B_{2}=0$. From Theorem 4 , we know that it is never optimal to set $B_{1}=\frac{\alpha_{L}^{2} r(2-r)}{2 k_{M}}$. This leaves one case for us to check:

$B_{1}=\frac{\alpha_{H}^{2} r(2-r)}{2 k_{M}}$ : From the first order condition we have $r^{*}=1$ as the only real root. Substituting this into $B_{1}=\frac{\alpha_{H}^{2} r(2-r)}{2 k_{M}}$ gives $B_{1}=\frac{\alpha_{H}^{2}}{2 k_{M}}$ and $B_{2}=0$. This is equivalent to an exclusion contract.

\section{Proof of Proposition 2}

1. (a) follows from Theorem 1 and (b) follows from Theorem 3

2. This follows from the innovator's optimal choice of research effort which is given by:

$$
\hat{e}=(1+\gamma) l+r(1-r)\left(\alpha_{H}^{2}+\gamma \alpha_{L}^{2}\right) /\left(2 k_{M}\right) \text {. }
$$

\section{Proof of Proposition 3}

The condition on renegotiation $F \leq \frac{\alpha_{L}^{2}}{2}\left(\frac{1}{k_{M}}-\frac{1}{k_{I}}\right)$ follows from the innovator's ex post incentive to initiate renegotiation in the low market-potential outcome, namely when the payoff after renegotiation, $\frac{\alpha_{L}^{2}}{2 k_{M}}-F$, is larger than the payoff without renegotiation, $\frac{\alpha_{L}^{2}}{2 k_{I}}$. 
The optimization then follows the same lines as the proof of Theorem 1 .

\section{Proof of Proposition 4}

The innovator's contract optimization problem is split into the case in which contracting under low market-potential is profitable, or $F_{2} \leq \alpha_{L}^{2} / 2\left(1 / k_{M}-1 / k_{I}\right)$, and otherwise. In both cases, the optimization follows the same lines as the proof of Theorem 1 .

\section{Proof of Proposition 5}

Following the solution procedure common for adverse selection problems we cover the interesting case where ICH and IRL bind (Bolton and Dewatripont (2005)), which leads to a separating equilibrium. None of the other cases lead to separating equilibria. We can solve the two binding constraints for the contract parameters $l_{H}$ and $l_{L}$. This gives us $l_{H}=\frac{\left(\left(2 r_{L}-r_{L}^{2}\right)-\left(2 r_{H}-r_{H}^{2}\right)\right) \alpha_{H}^{2}+\left(1-r_{L}\right)^{2} \alpha_{L}^{2}}{2 k_{M}}$ and $l_{L}=\frac{\left(1-r_{L}\right)^{2} \alpha_{L}^{2}}{2 k_{M}}$. We ignore the constraint ICL for the moment and check that it is satisfied at the end. Substituting expressions for $l_{H}$ and $l_{L}$ into the objective function and differentiating with respect to $r_{H}$ gives $\frac{\partial \Omega\left(e, r_{L}, r_{H}\right)}{\partial r_{H}}=-\frac{\pi_{H}(e) \alpha_{H} r_{H}}{k_{M}}$. Since $\pi_{H}(e)$ and $k_{M}$ are positive, and reducing $r_{H}$ relaxes $\mathrm{IRH}$, the only remaining constraint that is affected is $r_{H} \geq 0$. We can therefore set $r_{H}=0$. Substituting $r_{H}=0$ into the expression for $l_{H}$ gives

$$
l_{H}=\frac{\left(2 r_{L}-r_{L}^{2}\right) \alpha_{H}^{2}+\left(1-r_{L}\right)^{2} \alpha_{L}^{2}}{2 k_{M}}
$$

Substituting the expressions for other contract parameters into the objective function, differentiating the objective function w.r.t. $r_{L}$ and solving the FOC for $r_{L}$ we get

$$
r_{L}=\frac{\pi_{H}(e)\left(\alpha_{H}^{2}-\alpha_{L}^{2}\right)}{\pi_{H}(e)\left(\alpha_{H}^{2}-\alpha_{L}^{2}\right)+\pi_{L}(e) \alpha_{L}^{2}} .
$$

Since $\alpha_{H}>\alpha_{L}>0$ and $\pi_{L}(e), \pi_{H}(e) \geq 0$ with at least one probability strictly greater than zero, the expression for $r_{L}$ is always nonnegative. Substituting the above expressions for contract parameters into the ignored constraint ICL reveals that it is satisfied. This completes the proof.

\section{Proof of Theorem 6}

If the innovator were to offer a menu of contracts at $t=1$ the ICH and ICL constraints would remain the same as in Proposition 5 as they need to be satisfied at time $\mathrm{t}=4$. We denote them as $\mathrm{ICH}_{4}$ and $\mathrm{ICL}_{4}$. Similarly, we need constraints that ensure the product is launched at time $\mathrm{t}=4$, when the signature fee $s$ is sunk, and denote them by $\mathrm{IRL}_{4}$ and $\mathrm{IRH}_{4}$. We also need a constraint $\mathrm{IR}_{1}$ that makes sure that the marketer wants to participate at 
time $\mathrm{t}=1$. This gives us the optimization problem below.

$$
\begin{aligned}
\max _{0 \leq l_{i}, s, 0 \leq r_{i} \leq 1} & \Omega\left(e, l_{L}, l_{H}, r_{L}, r_{H}, s\right)=\pi_{L}(e)\left(l_{L}+\frac{r_{L}\left(1-r_{L}\right) \alpha_{L}^{2}}{k_{M}}\right)+\pi_{H}(e)\left(l_{H}+\frac{r_{H}\left(1-r_{H}\right) \alpha_{H}^{2}}{k_{M}}\right)+s \\
\text { s.t. } \quad & \frac{\left(1-r_{H}\right)^{2} \alpha_{H}^{2}}{2 k_{M}}-l_{H} \geq \frac{\left(1-r_{L}\right)^{2} \alpha_{H}^{2}}{2 k_{M}}-l_{L} \\
& \frac{\left(1-r_{L}\right)^{2} \alpha_{L}^{2}}{2 k_{M}}-l_{L} \geq \frac{\left(1-r_{H}\right)^{2} \alpha_{L}^{2}}{2 k_{M}}-l_{H} \\
& \pi_{H}(e)\left(\frac{\left(1-\mathrm{ICH}_{4}\right)}{2 k_{M} \alpha_{H}^{2}}-l_{H}\right) \geq 0 \\
& \pi_{L}(e)\left(\frac{\left(1-r_{L}\right)^{2} \alpha_{L}^{2}}{2 k_{M}}-l_{L}\right) \geq 0 \\
& \pi_{L}(e)\left(\frac{\left(1-\mathrm{ICL}_{4}\right)}{\left.2 k_{M}\right)^{2} \alpha_{L}^{2}}-l_{L}\right)+\pi_{H}(e)\left(\frac{\left(1-r_{H}\right)^{2} \alpha_{H}^{2}}{2 k_{M}}-l_{H}\right)-s \geq 0
\end{aligned}
$$

First, note that when $s=0$, the optimal solution in Proposition 5 clearly satisfies the constraints of this optimization problem. The first constraints remain the same. The first term of the $\operatorname{IR}_{1}$ constraint $\pi_{L}(e)\left(\frac{\left(1-r_{L}\right)^{2} \alpha_{L}^{2}}{2 k_{M}}-l_{L}\right)$ is equal to zero while the second term $\pi_{H}(e)\left(\frac{\left(1-r_{H}\right)^{2} \alpha_{H}^{2}}{2 k_{M}}-l_{H}\right)$ is strictly positive.

This shows that delayed contracting with unobservable market-potential can never outperform upfront contracting with unobservable market-potential.

Second, note that this solution can be replicated by the upfront buy-out contract $s=0$, $l=\frac{(1-r)^{2} \alpha_{L}^{2}}{2 k_{M}}$ and $r=\frac{\pi_{H}(e)\left(\alpha_{H}^{2}-\alpha_{L}^{2}\right)}{\pi_{H}(e)\left(\alpha_{H}^{2}-\alpha_{L}^{2}\right)+\pi_{L}(e) \alpha_{L}^{2}}$ and $B=l_{H}^{*}-l_{L}^{*}=\frac{\left(2 r-r^{2}\right) \alpha_{H}^{2}}{2 k_{M}}$. The L-type gets the same contract as Proposition 5. The $\mathrm{H}$ type gets an equivalent contract once the option is exercised.

Finally, as $I R_{1}$ is not binding in the above solution, increasing $s$ by a small increment $h>0$ clearly improves the maximand without affecting the first four constraints and still satisfying $I R_{1}$. The innovator can continue increasing $s$ and improving the maximand until $I R_{1}$ binds.

This shows that an upfront full contract with a buy-out option always outperforms both delayed contracting with unobservable market-potential and an upfront buy out option without an upfront payment. This completes the proof.

\section{References}

Aghion, P., P. Bolton. 1992. An incomplete contracts approach to financial contracting. The Review of Economic Studies 59(3) 473-494.

Aghion, P., J. Tirole. 1994. The management of innovation. The Quarterly Journal of Economics 109(4) 1185-1209. 
Alchian, A.A., H. Demsetz. 1972. Production, information costs, and economic organization. The American Economic Review 62(5) 777-795.

Anderson, Q. 2014. Licensing league tables 3Q14. Infinata Biopharm Insight .

Bhaskaran, S.R., V. Krishnan. 2009. Effort, revenue, and cost sharing mechanisms for collaborative new product development. Management Science 55(7) 1152-1169.

Bhattacharya, S., V. Gaba, S. Hasija. 2014. Coordinating bilateral investments in a healthcare R\&D partnership. Forthcoming in Management Science .

Bolton, P., M. Dewatripont. 2005. Contract Theory. The MIT Press.

Che, Y.K., D.B. Hausch. 1999. Cooperative investments and the value of contracting. American Economic Review 89 125-147.

Chung, T.Y. 1991. Incomplete contracts, specific investments, and risk sharing. The Review of Economic Studies 58(5) 1031-1042.

Dechenaux, E., M. Thursby, J. Thursby. 2009. Shirking, sharing risk and shelving: The role of university license contracts. International Journal of Industrial Organization 27(1) 80-91.

Edlin, A.S., S.J. Reichelstein. 1996. Holdups, standard breach remedies, and optimal investment. American Economic Review .

Elfenbein, D.W., J. Lerner. 2003. Ownership and control rights in internet portal alliances, 19951999. RAND Journal of Economics 356-369.

Gans, J.S., D.H. Hsu, S. Stern. 2008. The impact of uncertain intellectual property rights on the market for ideas: Evidence from patent grant delays. Management Science 54(5) 982-997.

Garleanu, N., J. Zwiebel. 2009. Design and renegotiation of debt covenants. Review of Financial Studies 22(2) 749-781.

Grossman, S.J., O.D. Hart. 1986. The costs and benefits of ownership: A theory of vertical and lateral integration. The Journal of Political Economy 691-719.

Hart, O., J. Moore. 1988. Incomplete contracts and renegotiation. Econometrica: Journal of the Econometric Society 755-785.

Hart, O., J. Moore. 1999. Foundations of incomplete contracts. Review of economic Studies 66(1) $115-138$.

Hellmann, T. 1998. The allocation of control rights in venture capital contracts. The Rand Journal of Economics 57-76.

Holden, S. 1999. Renegotiation and the efficiency of investments. The Rand Journal of Economics 106-119.

Kalamas, J., G. Pinkus. 2003. The optimum time for drug licensing. Nature Reviews Drug Discovery 2(9) 691-692.

Katila, R., P.Y. Mang. 2003. Exploiting technological opportunities: the timing of collaborations. Research policy 32(2) 317-332.

Lerner, J., U. Malmendier. 2010. Contractibility and the design of research agreements. American Economic Review 100(1) 214-246.

Lerner, J., R.P. Merges. 1998. The control of technology alliances: An empirical analysis of the biotechnology industry. The Journal of Industrial Economics 46(2) 125-156.

Nöldeke, G., K.M. Schmidt. 1995. Option contracts and renegotiation: a solution to the hold-up problem. The RAND Journal of Economics 163-179.

Pharma Deals Review. 2011. A review of deal making in 2010. (14 February). 
Plambeck, E.L., T.A. Taylor. 2007. Implications of renegotiation for optimal contract flexibility and investment. Management Science 53(12) 1872-1886.

Roijakkers, N., J. Hagedoorn. 2006. Inter-firm R\&D partnering in pharmaceutical biotechnology since 1975: Trends, patterns, and networks. Research Policy 35(3) 431-446.

Savva, N., S. Scholtes. 2013. Opt-out options in new product co-development partnerships. Forthcoming in Production and Operations Management .

Schuett, F. 2012. Field-of-use restrictions in licensing agreements. International Journal of Industrial Organization 30(5) 403-416.

Smirnov, V., A. Wait. 2004. Hold-up and sequential specific investments. RAND Journal of Economics 386-400.

Tirole, J. 1999. Incomplete contracts: Where do we stand? Econometrica 67(4) 741-781.

Xiao, W., Y. Xu. 2012. The impact of royalty contract revision in a multi-stage strategic R\&D alliance. Management Science 52(12). 